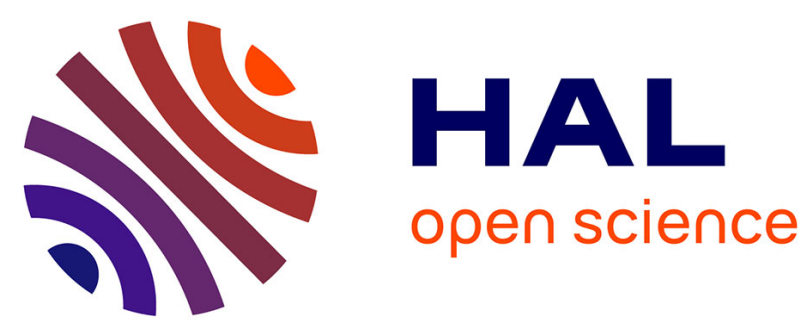

\title{
Ablation of carbon-based materials : investigation of roughness set-up from heterogeneous reactions
}

Georges Duffa, Gerard L. Vignoles, J.-M. Goyhénèche, Yvan Aspa

\section{To cite this version:}

Georges Duffa, Gerard L. Vignoles, J.-M. Goyhénèche, Yvan Aspa. Ablation of carbon-based materials : investigation of roughness set-up from heterogeneous reactions. International Journal of Heat and Mass Transfer, 2005, 48, pp.3387-3401. hal-00363237

\section{HAL Id: hal-00363237 \\ https://hal.science/hal-00363237}

Submitted on 20 Feb 2009

HAL is a multi-disciplinary open access archive for the deposit and dissemination of scientific research documents, whether they are published or not. The documents may come from teaching and research institutions in France or abroad, or from public or private research centers.
L'archive ouverte pluridisciplinaire HAL, est destinée au dépôt et à la diffusion de documents scientifiques de niveau recherche, publiés ou non, émanant des établissements d'enseignement et de recherche français ou étrangers, des laboratoires publics ou privés. 


\title{
Ablation of carbon-based materials : investigation of roughness set-up from heterogeneous reactions
}

\author{
Georges Duffa ${ }^{\text {b }}$, Gérard L. Vignoles ${ }^{\mathrm{a}, *}$, \\ Jean-Marc Goyhénèche ${ }^{\mathrm{a}}$, and Yvan Aspa ${ }^{\mathrm{a}, \mathrm{c}}$ \\ ${ }^{a}$ Lab. for ThermoStructural Composites (LCTS), UMR 5801 \\ CNRS-CEA-Snecma-Université Bordeaux l, 3, Allée La Boëtie, F-33600 Pessac, \\ France \\ ${ }^{\mathrm{b}}$ CEA-CESTA, BP2, F-33114 Le Barp, France \\ ${ }^{\mathrm{c}}$ Institute for Fluid Mechanics in Toulouse (IMFT), UMR 5502 \\ INPT-UPS-CNRS, 3 Allée du Pr. Camille Soula, F-31400 Toulouse, France
}

\begin{abstract}
Ablation of carbon-based materials is a key issue in atmospheric reentry ; it displays a strong coupling between mass, momentum and heat transfers, the importance of which relies on the surface roughness. A new possible physical cause for roughness set-up is investigated, based on the coupling between diffusive transfer in the surrounding fluid on one hand, and heterogeneous reaction or sublimation on the other. Considering mass transfer in a 2D, isothermal, vertical-flux approximation, the surface is proved to be able to acquire, among others, a stable stationary morphology made of circle arcs connected by symmetrical singular points. Such a morphology has indeed been observed in the case of graphite ablation, and the computed roughness length scale, arising from the diffusion-to-reaction ratio, is compatible with observed
\end{abstract}


data. A similar model based on the presence of a thermal gradient yields similar results, but with a larger length scale, also compatible with other observations.

* to whom correspondence should be addressed

Email addresses: gduffa@club-internet.fr (Georges Duffa),

vinhola@lcts.u-bordeaux.fr (Gérard L. Vignoles), goyhenec@lcts.u-bordeaux.fr (Jean-Marc Goyhénèche), yvan_aspa@yahoo.fr (Yvan Aspa). 


\section{Nomenclature}

Latin

$C=$ gas-phase concentration, $\mathrm{mol} \cdot \mathrm{m}^{-3}$

$\langle c\rangle \quad=\quad$ Root-mean-square molecular velocity, $m . s^{-1}$

$D \quad=$ diffusion coefficient,$m^{2} \cdot s^{-1}$

$D a=$ Damköhler number

$E_{a}=$ activation energy, J.mol ${ }^{-1}$

$\mathcal{H}=$ Hamiltonian operator, $m \cdot s^{-1}$

$h=$ height from upper domain limit, $m$

$h_{0} \quad=\quad$ reference height, $m$

$\Im m(x)=$ imaginary part of a complex number

$\mathbf{J} \quad=$ mole flux, $\mathrm{mol} \cdot \mathrm{m}^{-2} \cdot \mathrm{s}^{-1}$

$k=$ heterogeneous reaction coefficient, $m \cdot s^{-1}$

$\mathbf{k}=$ wave vector of a perturbation, $m^{-1}$

$\mathbf{M}=$ position of a surface point, $m$

$\mathcal{M}=$ molar mass, $\mathrm{kg} \cdot \mathrm{mol}^{-1}$ 


\section{List of Symbols (cont'd)}

$\mathbf{n}=$ normal vector of the interface

$p \quad=$ pressure, $\mathrm{Pa}$

$q=$ negative normal velocity amplitude, $m \cdot s^{-1}$

$q_{0} \quad=$ heat flux, $W \cdot m^{-2}$

$R=$ mole ablation rate per unit surface, $\mathrm{mol} \cdot \mathrm{m}^{-2} \cdot \mathrm{s}^{-1}$

$\Re e(x)=$ real part of a complex number

$S=$ function allowing to define the surface as an isosurface, $m$

$T=$ temperature, $K$

$t \quad=$ time, $s$

$\mathcal{V} \quad=$ global ablation velocity, $m \cdot s^{-1}$

$\mathcal{W}=$ velocity of a point of the surface, $m \cdot s^{-1}$

$x \quad=\quad$ lateral space coordinate, $m$

$y \quad=\quad$ lateral space coordinate, $m$

$z \quad=\quad$ vertical space coordinate, $m$ 


\section{List of Symbols (cont'd)}

Greek

$\alpha=$ sticking coefficient

$\Gamma_{\mathcal{W}}=$ graph of $\mathcal{W}$

$\gamma=$ Knudsen-Langmuir parameter

$\gamma_{a}=$ scaled activation energy

$\gamma_{f s}=$ fluid-solid interface energy, J.m $m^{-2}$

$\delta \quad=$ perturbation

$\eta \quad=$ position of the diffusive boundary layer top , $m$

$\kappa=$ curvature, $m^{-1}$

$\lambda=$ dimensionless width

$\lambda_{0}=$ thermal conductivity, $W \cdot m^{-1} \cdot K^{-1}$

$\nu \quad=$ stoichiometric factor

$\Sigma \quad=$ segment

$\theta=$ tilt angle of the surface at current point, $\mathrm{rad}$

$\theta_{0}=$ critical tilt angle for $\Gamma_{\mathcal{W}}$, rad

$\tau \quad=$ time scale factor, $s$

$v \quad=$ molar volume, $m^{3} \cdot m o l^{-1}$ 


\section{List of Symbols (cont'd)}

$\phi=$ angle between the normal to the surface and the flux, rad

$\omega=$ time pulsation of a perturbation, $s^{-1}$

Subscripts and superscripts

$\boldsymbol{~}_{g} \quad=$ relative to a gas-phase species,

$\boldsymbol{\bullet}_{s} \quad=$ relative to a solid phase,

$\bullet_{0} \quad=$ reference quantity,

$\bullet_{+}=$relative to the right-hand side of a singular point

- $\quad=$ relative to the left-hand side of a singular point

$\tilde{\bullet}=$ dimensionless variable

$\bullet \quad=$ relative to a moving coordinate frame

- $\quad=$ relative to equilibrium or stationarity

Constants

$k_{B}=1.3806581 \cdot 10^{-23} \mathrm{~J} \cdot K^{-1}:$ Boltzmann's constant

$\mathcal{N}=6.02213674 \cdot 10^{23}$ mole $e^{-1}:$ Avogadro's number

$\mathcal{R}=8.3145107 \mathrm{~J} \cdot$ mole $^{-1} \cdot K^{-1}:$ perfect gas constant 


\section{Key words}

- Ablation

- Sublimation

- Heterogeneous reaction

- Diffusion

- Roughness

- Coupling

- Hamilton-Jacobi equation

\section{Introduction}

Carbon/carbon $(C / C)$ composites are the almost unique class of thermostructural materials that are used as thermal protection of atmospheric reentry bodies. Indeed, the local conditions are dramatically severe : temperatures up to $4500 \mathrm{~K}$ and pressures ranging between 0.1 and 100 bars, and a heat flux received by the protection ranging from 0.1 to $500 M W \cdot m^{-2}$. In such conditions, a non-negligible part of the heat flux is consumed by interfacial mass transfer, which has two principal forms : oxidation (and other chemical reactions) and sublimation. These phenomena are grouped under the generic name of ablation [1]. Their particularity is to display a strong coupling with many other physical phenomena, namely fluid flow and heat transfer.

The surface roughness related to the ablation phenomena of the atmospheric reentry body thermal protections has considerable consequences. It is able to promote a laminarto-turbulent regime transition [2,3], a fact which induces a strong enhancement of heat and mass exchanges between the protection wall and the surrounding environment. It has been experimentally observed that the heat flux may be multiplied by a factor up to three 
in turbulent regime [4]. The consequences on the surface temperature and heat-affected zone depth are obvious, as well as on the overall ablation velocity.

However, little is known of the creation mechanisms of such a surface state, which results from a strong coupling between the material and the surrounding flow. The aim of this work is to set up and use simple models of interaction, involving the least possible phenomena at first, that is, merely the heterogeneous reaction and mass transport from the flowing fluid.

The first question that one can ask about material/fluid interaction is : what is the relation between the surface morphology and the underlying physico-chemical phenomena ? This question has been dealt with in numerous works on CVD (Chemical Vapor Deposition) [5-9], which is closely related to ablation, the difference being the sign of the wall velocity. Palmer and Gordon[6,7] has found out by linear perturbation theory and by numerical simulation that at flat surface may be destabilized by gas-phase diffusion tangent to the surface, while surface diffusion had the opposite effect. Another analytical approach [8] focused on the modification of the local growth rate due to curvature, and a competition of such an effect with surface diffusion : the result is that wavy surface may develop. Thiart, Hlavaček, and Viljoen [9] have developed efficient numerical tools for the simulation of CVD growth in various conditions and have shown that "fingering" appears in the case of diffusion-limited growth, a fact that has been confirmed by linear stability analysis $[5,9]$.

In the case of graphite ablation, experimental determinations of the roughness have been performed by profilometry, using well-defined flow conditions and heat transfer rates [10]. In some experimental conditions for which the flow is laminar (Fig. 1a), the surface of a polycrystalline graphite looks smooth up to a few $\mu m$ resolution. The apparent roughness seems to rely only on the material heterogeneity, which is known to contain small domains of better cristallinity than the surrounding matter. Roughness here may only be the 
result of the material heterogeneity (variations of the density and local heterogeneous kinetic constant) : this issue will be addressed in the following parts, mostly in the case of carbon/carbon $(C / C)$ composites, which are made of different types of carbon. An example is given at fig. 2: in a composite the matrix of which is made of a very lowdensity (and high-reactivity) carbon, the fibers, which are more resistant, are salient with respect to the matrix, and acquire an ogival shape. In a composite, the space scales are related to the individual fiber diameter (e.g. $\approx 7 \mu \mathrm{m}$ for carbon fibers), and to the apparent diameter of tows, warps, needlings, etc...(e.g. $\approx 300-500 \mu m$ for many composites).

Nonetheless, in turbulent flow conditions, the surface of the same material displays a "cellular" roughness (fig. 1b) with length scales (here, roughly $1 \mathrm{~mm}$ ) that cannot be related to the material heterogeneity nor to turbulent length scales. This kind of "scalloped" surface is strikingly similar to glacier cave walls. In addition, a closer look at the fibers in the ablated $C / C$ composite shown at fig . 2 reveals other scalloped features on single composite fibers, this time with a length scale around $10 \mu m$, that are clearly seen in fig. 3. Again, it cannot be related to the material heterogeneity ; so the explanation of this has to arise from an extrinsic phenomenon.

It will be tried to show that natural length scales arising from the coupling of transport and heterogeneous reaction are of comparable orders of magnitude, since other solutions than the trivial flat surface can be produced from a simple model. Most of the development presented will be devoted to mass transfer by diffusion, and heat transfer will be treated by analogy.

It is worthwhile to note that although CVD growth morphologies are sometimes similar to these ablation morphologies (though with opposite concavities), they can not be accounted for with the same kind of model.

In the following part, a diffusion-reaction model will be recalled ; then an evolution equa- 
tion will be produced for the surface points. Stationary solutions will be presented in the case of a growing boundary layer (initial transient state) and in the case of a constant boundary layer (steady-state) and some discussions on stability will be made. Singular points will be next considered, and a condition for their stability will be worked out. Numerical simulations will then be presented, which confirm the steady-state analysis and give an insight into the transient regimes. Then, an application of the model will be presented and discussed with respect to the available experimental facts. Finally, a brief presentation of a heat-transfer counterpart of the model is given, and the results are discussed in terms of shape and scale.

\section{Model set-up}

\subsection{Model frame and assumptions}

Let us consider a parallelepipedic domain $(x, y, z)$ containing a part of the fluid, and a part of the solid (fig. 4). The interface (or surface) is globally perpendicular to the $z$ direction and may be described by a set of points $(x, y, z)$ such that $S(x, y, z, t)=0[11$. Indeed, a particular case of this general formulation will be used frequently, when $S$ may be explicited under the form $S=h(x, y, t)-z$. The $S=0$ condition coincides then with the definition of a unique height $h$ at which the interface lies. In this case, $S$ has the dimension of a length ; however, other choices are possible for such a "potential" function : for example, the local proportion of solid could be an acceptable candidate for $S$, and the formulation would be a VOF (Volume of Fluid) formulation [12]. The fluid lies in the upper part of the domain, with $z$ pointing downwards. The height of the diffusion path $h$ is assumed to have an order of magnitude at least comparable to the roughness characteristic length. The $(x, y)$ extension of the domain may be much larger than the $z$ extension. If 
needed, periodic boundary conditions may be defined in the $x$ and $y$ directions. See fig. 4 for a $2 \mathrm{D}$ scheme. For most purposes of this work, only two dimensions of space will be needed.

The fluid transfer will be limited to pure diffusion. The chemical reactants will be considered as having a fixed, prescribed concentration either at the top of the domain $(z=0)$ or at a given height $z=\eta(t)$, and diffusing downwards, while the species created by the ablation process diffuse upwards and their concentration at the upper boundary will be held at zero value. This represents indeed the fact that convection is non-negligible outside of the studied domain, and that $h$ is also the size of the diffusive boundary layer $D / v_{\text {conv }}$. Two physical cases in which this is a valid assumption may be quoted :

(1) In real reentry conditions, the height of this boundary layer is very small since the convective velocity is extremely high ; however, a stagnation point has to exist at the top of the reentering object, with moderate convection in its vicinity. In such a region, the size of the diffusive layer may be larger.

(2) In laboratory experiments, the convective velocity is moderate - and even low - at some parts of the ablated sample, especially in "stop-point" configurations.

It is not yet possible to perform full calculations featuring the whole physics of atmospheric reentry (i.e. including turbulence with Kolmogorov scale around $10 \mu \mathrm{m}$ ) and covering a typical macroscopic cell $($ size $\approx 1 \mathrm{~cm})$. These two length scales are such that any DNS simulation is not feasible ; and since no data is available on the precise effect of surface roughness on turbulence, it is not possible either to perform LES simulations. Nonetheless, experimental evidence shows that the surface morphology is not very different below the stagnation point (for which it is acceptable to neglect convection) and at the rear part of the reentering body (with Mach numbers superior to one outside the viscous boundary layer). This suggests that a first approach not featuring convection may have some interest. 
Stefan flux, that is, the normal mass flux entering the gas phase at the wall, will be neglected for two reasons :

(1) In many practical cases, it may be considered that the gaseous molecules involved in reaction and/or sublimation are diluted in a carrier gas.

(2) An experimental study of the modification of heat transfer under the effect of gas blowing from a porous substrate [13] shows that the transfer rate is altered by at most $10 \%$ when realistic values for the blowing/input fluxes are chosen. If Lewis and Prandtl numbers are close to unity, it is straightforward to extend this result to mass transfer, that is : Stefan flux alters by less than $10 \%$ the total flux amount in any case of interest. We may thus neglect it safely in a first approach.

The fluid domain will be considered as isobaric and isothermal. This is not a strong assumption in the case of a small domain height ; the converse case will be studied in another paper. As a consequence, the transport and reaction properties will be considered constant throughout the domain.

Curvature and surface diffusion effects will be neglected at first in this approach, although they are known to have important effects in CVD. However, it will be seen that they may only be seen as second-order corrections to the principal results of the present study.

\subsection{Surface evolution}

The surface having a local normal velocity $\mathbf{v}$, its time and space evolution obeys the following equation at all points where $S$ is differentiable [11]:

$$
\frac{\partial S}{\partial t}+\mathbf{v} \cdot \nabla S=0
$$


This equation only means that in a local coordinate frame moving with velocity $\mathbf{v}$, the implicit equation defining the surface is preserved. The expression of the velocity relies on the ablation phenomenon ; thus, it depends on the ablation molar rate $R$. Its expression follows from a simple mass balance equation :

$$
\mathbf{v}=v_{s} R \mathbf{n}
$$

where $v_{s}$ is the solid molar volume and the normal vector is defined as :

$$
\mathbf{n}=\frac{\nabla S}{\|\nabla S\|}
$$

By convention, it will be pointing towards the fluid ; as a consequence, the quantity $S$ will be larger in the fluid than in the solid. The ablation molar rate may be related to the impinging mole flux $\mathbf{J}$ :

$$
R=\left(\frac{\nu_{s}}{\nu_{g}}\right) \mathbf{J} \cdot \mathbf{n}
$$

where the term between parentheses represents the stoichiometric ratio of ablated solid with respect to the gaseous species. For a chemical reaction (or a phase change) noted $\sum_{i} \nu_{i} A_{i}=0$, the stoichiometric coefficients $\nu_{i}$ will be counted negative for the reactants and positive for the products. They are detailed at table 1. Two cases are distinguished : i) a first-order reaction with an etching species, like $\mathrm{O}_{2}$ for example, and $i i$ ) spontaneous sublimation. The case of CVD is given as a comparison.

Collecting eqs. (1), (2), and (4) together, one has the following equation for $S(x, y, z, t)$ :

$$
\frac{\partial S}{\partial t}=-v_{s}\left(\frac{\nu_{s}}{\nu_{g}}\right)(\mathbf{J} \cdot \nabla S)
$$

Under the considered approximations, the gas mole flux is given by Fick's first law :

$$
\mathbf{0}=\mathbf{J}+D \nabla C
$$


and the mass conservation in the gas phase reads :

$$
\frac{\partial C}{\partial t}=-\nabla \cdot \mathbf{J}
$$

Let us now define a reference height $h_{0}$. This may be for instance the size of a diffusion boundary layer, resulting from the interaction of ablation and of an outer tangent gas stream. Let also $C_{0}$ be a reference gas-phase concentration. The variables may be scaled in the following form :

$$
\begin{gathered}
x=h_{0} \tilde{x} \quad C=C_{0} \tilde{C} \quad S=h_{0} \tilde{S} \\
\nabla=h_{0}^{-1} \tilde{\nabla} \quad t=\frac{h_{0}^{2}}{D C_{0} v_{s}} \tilde{t} \quad \mathbf{J}=\frac{D C_{0}}{h_{0}} \tilde{\mathbf{J}}
\end{gathered}
$$

Then, the equations to solve are :

$$
\left\{\begin{aligned}
\left(v_{s} C_{0}\right) \frac{\partial \tilde{C}}{\partial \tilde{t}} & =-\tilde{\nabla} \cdot \tilde{\mathbf{J}} \\
\mathbf{0} & =\tilde{\mathbf{J}}+\tilde{\nabla} \tilde{C} \\
\frac{\partial \tilde{S}}{\partial \tilde{t}} & =-\left(\frac{\nu_{s}}{\nu_{g}}\right) \tilde{\mathbf{J}} \cdot \tilde{\nabla} \tilde{S}
\end{aligned}\right.
$$

Since the dimensionless group $v_{s} C_{0}$ (the condensation ratio) is a small quantity, the first term in the first equation may be considered as zero - that is, gas diffusion being rapid with respect to surface evolution, it can be considered in steady state. Dropping out all tildes for sake of simplicity, one now has the following set of equations :

$$
\left\{\begin{aligned}
\nabla^{2} C & =0 \\
\frac{\partial S}{\partial t} & =+\left(\frac{\nu_{s}}{\nu_{g}}\right) \nabla C \cdot \nabla S
\end{aligned}\right.
$$


subject to boundary conditions sets which are linked to the precise nature of the reaction or change of state.

\subsection{Boundary conditions and mass sources}

The boundary conditions are listed at Table 1. Two limiting cases are considered. Indeed, it is well known that carbon loss in atmospheric reentry arises principally from two phenomena : $i$ ) oxidation or similar reaction (with nitrogen for example), and $i i$ ) carbon sublimation. The first case may have complicated kinetic laws, the apparent order of which ranges usually between 0 and 1 [14]. The limiting case of first-order wall reaction will be dealt with first ; in a second time, a local equilibrium model, applicable either to sublimation or to a weak chemical reaction, will be presented.

For a first-order irreversible reaction, it is considered that the concentration is held fixed at a constant value $C_{0}$ - which is used as a reference for dimensionless quantities - at the boundary layer top, which lies at some height $\eta(t)$, and is considered as flat.

The boundary conditions are :

$$
\left\{\begin{array}{c}
C(z=\eta)=1 \\
-\nabla C \cdot \nabla S=\nu_{g} D a\|\nabla S\| C \text { at } S=0
\end{array}\right.
$$

where the Damköhler number has been introduced : $D a=\frac{k h_{0}}{D}$, with $k$ being a first-order heterogeneous reaction constant in $m . s^{-1}$. The stoichiometric coefficients $\nu_{s}$ and $\nu_{g}$ are both negative in the case of oxidation. For example, for the reaction

$$
C(s)+\mathrm{CO}_{2}(g) \longrightarrow 2 \mathrm{CO}(g)
$$


, we have $\nu_{g}=\nu\left(C O_{2}\right)=\nu_{s}=-1$. The CVD reaction

$$
\mathrm{CH}_{4}(g) \longrightarrow \mathrm{C}(s)+2 \mathrm{H}_{2}(g)
$$

yields $\nu_{s}=+1$ and $\nu_{g}=-1$.

In the case of sublimation, the species partial pressure is supposed to be zero at the upper limit of the domain. The net sublimation mole flux (in a direction normal to the wall) is given by the Knudsen-Langmuir relationship [15] in dimensional form :

$$
\mathbf{J} \cdot \mathbf{n}=\gamma(\bar{p}-p)
$$

where $\bar{p}$ is the equilibrium partial pressure and :

$$
\gamma=\frac{\alpha}{4} \frac{1}{\mathcal{R} T} \sqrt{\frac{8 \mathcal{R} T}{\pi \mathcal{M}}}
$$

Here, $\alpha$ is a sticking coefficient ${ }^{1}$. The term in square root is the root mean square molecular velocity $\langle c\rangle=\sqrt{\frac{8 \mathcal{R} T}{\pi \mathcal{M}}}$.

By comparison with the preceding case, and replacing the partial pressure $p$ by $C \mathcal{R} T$ from the perfect gas law, the sublimation flux is rewritten :

$$
\mathbf{J} \cdot \mathbf{n}=k(\bar{C}-C)
$$

where it has been possible to define an "equivalent kinetic constant" :

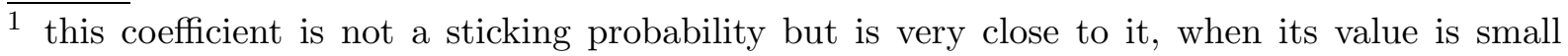
compared to 1 [15]. 


$$
k=\frac{\alpha}{4}\langle c\rangle
$$

Summarizing in dimensionless form, one has :

$$
\left\{\begin{array}{c}
C(z=\eta)=0 \\
-\nabla C \cdot \nabla S=\nu_{g} D a\|\nabla S\|(1-C) \text { at } S=0
\end{array}\right.
$$

In such a case, the stoichiometric coefficients are such that $\nu_{g}$ is positive while $\nu_{s}$ is negative. For example,

$$
3 C(s) \longrightarrow C_{3}(g)
$$

gives $\nu_{s}=-3$ and $\nu_{g}=+1$. In eqs. (16) it is assumed that the reference concentration $C_{0}$ is the equilibrium gas concentration $\bar{C}$ obtained from the Clausius-Clapeyron relation.

\subsection{Surface height as a function of the abscissa}

Now if the surface is such that $z$ can be solved unequivocally out of $S(x, y, z, t)=0$, that is, if :

$$
S(x, y, z, t)=0=h(x, y, t)-z
$$

then the evolution equation (5) for $S$ may be rewritten for the height $h$ of the surface :

$$
\frac{\partial h}{\partial t}+\left(\frac{\nu_{s}}{\nu_{g}}\right)\left(J_{x} \frac{\partial h}{\partial x}+J_{y} \frac{\partial h}{\partial y}-J_{z}\right)=0
$$


The normal vector is :

$$
\mathbf{n}=\frac{1}{\sqrt{1+\left(\frac{\partial h}{\partial x}\right)^{2}+\left(\frac{\partial h}{\partial y}\right)^{2}}}\left(\begin{array}{c}
\partial h / \partial x \\
\partial h / \partial y \\
-1
\end{array}\right)
$$

and points towards the fluid. The normal flux is then :

$$
\mathbf{J} . \mathbf{n}=\frac{1}{\sqrt{1+\left(\frac{\partial h}{\partial x}\right)^{2}+\left(\frac{\partial h}{\partial y}\right)^{2}}}\left(J_{x} \frac{\partial h}{\partial x}+J_{y} \frac{\partial h}{\partial y}-J_{z}\right)
$$

The boundary conditions (11) and (16) rewrite then :

$$
\left\{\begin{aligned}
C(z=\eta) & =1 \\
\left(J_{x} \frac{\partial h}{\partial x}+J_{y} \frac{\partial h}{\partial y}-J_{z}\right) & =\nu_{g} D a \sqrt{1+\left(\frac{\partial h}{\partial x}\right)^{2}+\left(\frac{\partial h}{\partial y}\right)^{2}} C \text { at } S=0
\end{aligned}\right.
$$

and :

$$
\left\{\begin{aligned}
C(z=\eta) & =0 \\
\left(J_{x} \frac{\partial h}{\partial x}+J_{y} \frac{\partial h}{\partial y}-J_{z}\right) & =\nu_{g} D a \sqrt{1+\left(\frac{\partial h}{\partial x}\right)^{2}+\left(\frac{\partial h}{\partial y}\right)^{2}}(1-C) \text { at } S=0
\end{aligned}\right.
$$

\subsection{Vertical flux approximation}

Let us now consider that the gaseous mole flux is vertical, that is, $\left|J_{x}\right|<<\left|J_{z}\right|$ and $\left|J_{y}\right|<<\left|J_{z}\right|$. In such a case, it is possible to reduce the system to only one equation, provided that the surface is not too steep, that is :

$$
\left|\frac{\partial h}{\partial i}\right|<<\left|\frac{J_{z}}{J_{i}}\right|, i=x, y
$$


For instance, if one chooses a slope 100 times inferior to a $\left|\frac{J_{z}}{J_{i}}\right|$ ratio, and a value of 100 for such a ratio, one obtains a critical surface inclination of $45^{\circ}$.

From Fick's laws, it appears that $J_{z}$ is a constant :

$$
J_{z}=-\frac{C(z)-C(\eta)}{z-\eta}=\mathrm{constant} \quad \forall x, y, z
$$

and that $C(x, y, z)=J_{z} z+$ constant. The boundary conditions (21) rewrite :

$$
\left\{\begin{array}{c}
C(\eta)=1 \\
C(h)-1=\nu_{g}(h-\eta) \cdot \text { Da.C }(h) \sqrt{1+\left(\frac{\partial h}{\partial x}\right)^{2}+\left(\frac{\partial h}{\partial y}\right)^{2}}
\end{array}\right.
$$

The concentration at the interface is then :

$$
C(h)=\frac{1}{1-(h-\eta) \nu_{g} D a \sqrt{1+\left(\frac{\partial h}{\partial x}\right)^{2}+\left(\frac{\partial h}{\partial y}\right)^{2}}}
$$

Substituting back into eqs. (18-21), one arrives at the following equation for $h$ at the interface $(z=h)$ :

$$
\frac{\partial h}{\partial t}=\left(\frac{\nu_{s}}{\nu_{g}}\right) \frac{1}{h-\eta-\frac{1}{\nu_{g} D a \sqrt{1+\left(\frac{\partial h}{\partial x}\right)^{2}+\left(\frac{\partial h}{\partial y}\right)^{2}}}}
$$

Or, letting $h^{\prime}=h-\eta$ and $\nu_{g}=\nu_{s}=-1$ :

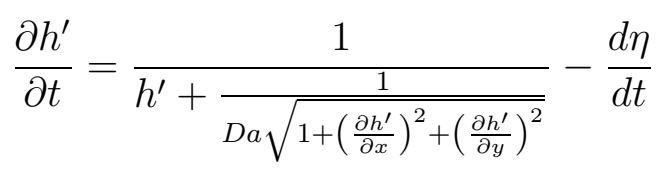

However, $d \eta / d t$ may be viewed as a mean ablation velocity, so it is interesting to extract from eq. (28) the local information. Choosing a reference frame with origin at $z=\eta$ and 
moving with velocity $d \eta / d t\left(i . e . x^{\prime}=x-t d \eta / d t ; t^{\prime}=t\right)$, one has :

$$
\frac{\partial h^{\prime}}{\partial t^{\prime}}=\left(h^{\prime}+\left(D a \sqrt{1+\left(\frac{\partial h^{\prime}}{\partial x^{\prime}}\right)^{2}+\left(\frac{\partial h^{\prime}}{\partial y^{\prime}}\right)^{2}}\right)^{-1}\right)^{-1}
$$

This is a Hamilton-Jacobi equation for $h^{\prime}$. In the case of sublimation, things are similar. The boundary conditions (22) may be rewritten :

$$
\left\{\begin{array}{c}
C(\eta)=0 \\
C(h)-1=\nu_{g}(h-\eta) \cdot D a \cdot(1-C(h)) \sqrt{1+\left(\frac{\partial h}{\partial x}\right)^{2}+\left(\frac{\partial h}{\partial y}\right)^{2}}
\end{array}\right.
$$

Making the same algebra as in the preceding case leads to the same equation as eq. (29) but with a reversed sign ; however, the stoichiometric ratio is now negative, so the result is :

$$
\frac{\partial h^{\prime}}{\partial t^{\prime}}=\left|\frac{\nu_{s}}{\nu_{g}}\right|\left(h^{\prime}+\left(\left|\nu_{g}\right| D a \sqrt{1+\left(\frac{\partial h^{\prime}}{\partial x^{\prime}}\right)^{2}+\left(\frac{\partial h^{\prime}}{\partial y^{\prime}}\right)^{2}}\right)^{-1}\right)^{-1}
$$

and is formally equal to the preceding one.

The obtained equation differs from previously proposed equations $[8,9]$ for several reasons

- The vertical flux in eq. (9) is not treated as an externally provided constant, but as dependent on the boundary layer height $h$ and on the interface concentration $C(h)$, which has indeed to be done in the case where neither diffusion nor reaction overcomes on each other ;

- The variation of the kinetic constant $k$ with the local curvature has been neglected ;

- The effect of surface diffusion, also sensitive to curvature, has been neglected. 


\section{$3 \quad$ Stationary profiles and velocities}

Equation (29) is indeed a Hamilton-Jacobi equation, for which "stationary solutions" means solutions with a fixed velocity in global frame, or with null velocity in local frame with respect to $d \eta / d t$.

Two distinct methods will be presented for the derivation of such solutions. First, a global criterion, based on the existence of a "prime integral" is used. Second, a local criterion based on the time stability of the slope (or the equality of the velocity for all points) will be used.

\subsection{Global (geometrical) method}

Let us introduce the angle $\varphi$ such that $\mathbf{J} \cdot \mathbf{n}=\|\mathbf{J}\|\|\mathbf{n}\| \cos \varphi$. Since we are in the hypothesis that $\mathbf{J}$ is strictly vertical, then :

$$
\cos \varphi=-\|\mathbf{n}\|^{-1}=-\left(\sqrt{1+\left(\frac{\partial h^{\prime}}{\partial x^{\prime}}\right)^{2}+\left(\frac{\partial h^{\prime}}{\partial y^{\prime}}\right)^{2}}\right)^{-1}
$$

In two dimensions, that is, dropping out the $y$ coordinate, from fig. 4 it is easy to see that

$\theta=\pi-\varphi=\arctan \frac{\partial h^{\prime}}{\partial x}$ is the angle between the slope of the interface and the horizontal axis. Introducing this angle into eq. (28) yields in fixed coordinates :

$$
\frac{\partial h^{\prime}}{\partial t}=\left(h^{\prime}+\frac{\cos \theta}{D a}\right)^{-1}-\frac{\partial \eta}{\partial t}
$$

where $\cos \theta$ is positive, i.e. $\theta \in(-\pi / 2 ; \pi / 2)$.

Then, stationarity of the interface $\left(\frac{\partial h^{\prime}}{\partial t}=0\right)$ implies conservation of the quantity : 


$$
h^{\prime}+D a^{-1} \cos \theta=1 / \mathcal{V}
$$

where $\mathcal{V}=\frac{\partial \eta}{\partial t}$ is the global velocity.

A trivial solution is $\theta=0$ everywhere, that is, a flat interface. A natural choice for the reference length $h_{0}$ is then such that $h^{\prime}=1$ when $\cos \theta=1$, i.e. at the surface. The velocity is then $\mathcal{V}=\frac{D a}{1+D a}$, that is, turning back to dimensional quantities :

$$
\frac{d \eta}{d t}=\frac{1}{D a+1} \cdot \frac{\nu_{s}}{\nu_{g}} \cdot\left(v_{s} C_{0}\right) \cdot k
$$

This choice for characteristic dimensions will be retained also in the following.

On the other hand, since $\frac{\partial h^{\prime}}{\partial x}=\tan \theta$, using a chain derivation rule, one obtains that $\frac{\partial x}{\partial \theta}=-D a^{-1} \cos \theta$. Then, by integration, $x=x_{0}+D a^{-1} \sin \theta$, and the solution is thus a circle arc with center at $\left(x_{0}, 1-1 / D a\right)$ and radius $1 / D a$ :

$$
\left\{\begin{array}{l}
h^{\prime}=1+D a^{-1}(1-\cos \theta) \\
x=x_{0}+D a^{-1} \sin \theta
\end{array}\right.
$$

Since $\cos \theta$ is positive, only the circle arc with a center lying in the fluid is correct. When $\theta=0$, that is, for a point with null slope, the velocity is equal to the flat-surface value, but is also equal to the velocity of the other points ; thus, the circle-arc solution has the same velocity as the flat one.

The analytic form of the solution is, in the moving coordinate frame :

$$
h^{\prime}(x)=-\sqrt{1-\left(x-x_{0}\right)^{2}}+h_{0}+1
$$


It is obvious that an interface with indefinite $x$ extension cannot be obtained from continuous solutions of eq. 28. This issue is readily dealt with, considering that a solid surface does not have to be smooth everywhere : it just has to be continuous. We will address further this point at next section ; however, a glance at fig. 6 helps to understand the shape of a particular, non-trivial solution.

\subsection{Local criterion}

Another way of working out stationary solutions is to consider directly eq. 28 (neglecting the $y$ coordinate for simplicity), derive it with respect to $x$, and equal the result to zero, which means that the slope does not vary with time and that the time evolution for all points is the same :

$$
\frac{\partial^{2} h}{\partial t \partial x}=0=-\left(\frac{\partial h}{\partial t}\right)^{2}\left(\frac{\partial h}{\partial x}\right)\left(1+D a^{-1} \kappa\right)
$$

where $\kappa$ is the curvature :

$$
\kappa=-\frac{\partial^{2} h / \partial x^{2}}{\left(1+(\partial h / \partial x)^{2}\right)^{3 / 2}}
$$

Stationarity of the profile is thus equivalent to having a constant curvature equal to $-D a$, i.e, a circle arc with a center lying in the fluid and radius $1 / D a$. This is exactly the same result as before.

\subsection{Singular points}

As it stands clear from fig. 1b), a description of the surface involving singular points seems to make enough sense. Moreover, the result of the preceding section is that a stationary continuous surface may be a circle arc ; thus, an interface with a large lateral extension may be described as a collection of circle arcs, at the expense of the existence of a certain 
number of singular points. Let us assume that there exists an enumerable quantity of such points (in practice it will be a finite quantity), then, since the surface is continuous, it is possible to define for every point of it a left-hand slope and a right-hand slope :

$$
\begin{aligned}
& \tan \theta_{+}(x)=\left.\frac{\partial h}{\partial \xi}\right|_{\substack{\xi \rightarrow x \\
\xi \geq x}} \tan _{-}(x)=\left.\frac{\partial h}{\partial \xi}\right|_{\substack{\xi \rightarrow x \\
\xi \leq x}}
\end{aligned}
$$

The solid mass balance (eq. 33) gives now two equalities, one for each "half-slope" :

$$
\frac{\partial h^{\prime}}{\partial t}=\left(h^{\prime}+\frac{\cos \theta_{+}}{D a}\right)^{-1}=\left(h^{\prime}+\frac{\cos \theta_{-}}{D a}\right)^{-1}
$$

From these relations, it is readily seen that two kinds of points may exist :

- $\theta_{+}=\theta_{-}$, that is, the surface is continuous at $x$;

- $\theta_{+}=\pi-\theta_{-}$, that is, there exists a singular point, and the curve is locally symmetrical with respect to a vertical axis at $x$.

It is also straightforward to see from the conservation relation (34) that all circle arcs have to display the same curvature if the material is homogeneous. Fig. 5 is a sketch of a typical possible solution, for which $i$ ) the linear density of singular points is $\mathcal{O}(D a)$, and $i$ ) most of the circle arcs contain a point with $\theta=0$. An exception to statement $i i$ ) is shown as a grey-filled circle : it is a reentrant singularity. It will be shown in a later section that such a singularity is not likely to be realized in practice.

Indeed, the experimentally observed patterns are such that the properties mentioned above hold. Why is it so ? Some qualitative arguments may be exposed. First, the linear density of singular points has to be superior or equal to $(2 D a)^{-1}$, since the individual circle arc definition range is at most $2 D a$. Second, if the linear density is much superior 
to $D a^{-1}$, then the curve becomes apparently smoother (i.e. the peak-to-valley parameter $[2,4]$ diminishes strongly).

Phenomena that have not been taken into account, like surface diffusion, curvatureinduced reactivity excess, or - more probably - thermal effects and deviation from the verticality of the diffusion fluxes, etc... are believed to smooth locally the surface and transform a singular point into a region with finite but strong curvature and width $\lambda<<D a$. The presented model is thus not applicable when the density of singular points becomes comparable or superior to $\lambda^{-1}$.

\section{Profile stability discussion}

This discussion will be split in two distinct parts : first, the smooth parts of the solution curves are studied through a standard perturbation calculus. Then, the stability of singular points will be treated using Huyghens' principle $[11,16]$.

\subsection{Continuous parts of the surface}

A standard stability analysis may be performed on the $2 \mathrm{D}$ stationary profile solutions $z=\bar{h}(x, t)$, by introducing perturbations of $C$ and $h$ :

$$
\begin{aligned}
& \Delta C=C-\bar{C}=\delta_{C} \exp \left(\omega_{C} t+k_{C, x} x+k_{C, z} z\right) \\
& \Delta h=h-\bar{h}=\delta_{h} \exp \left(\omega_{h} t+k_{h, x} x\right)
\end{aligned}
$$

The time and space pulsations $\omega_{i}$ and $k_{i}$ will be related by dispersion relations, after having checked their interdependency. Introducing the perturbation $\Delta C$ into Fick's second law 
yields :

$$
k_{C, x}^{2}+k_{C, z}^{2}=\left(v_{s} C_{0}\right) \omega_{C} \approx 0
$$

A correct choice for a perturbation should be with a decreasing amplitude for decreasing $h-z$, so that $k_{C, z}$ should be real and positive. As a consequence, $k_{C, x}= \pm j k_{C, z}$ is purely imaginary, i.e. the perturbation is periodic in $x$ direction. Using now eq. (18) yields :

$$
\omega_{h} \Delta h=\left(\bar{h}_{x} k_{C, x}-k_{C, z}\right) \Delta C
$$

where $\bar{h}_{x}=\partial \bar{h} / \partial x$ is a short notation for the local slope. This result, valid for all $x$, implies that $\Delta C$ and $\Delta h$ do have equal space periods :

$$
\begin{aligned}
& k_{h, x}=k_{C, x}=k_{x} \\
& k_{C, z}=k_{z}
\end{aligned}
$$

On the other hand, making use of eqs. (21) or (22) gives :

$$
-\bar{C}_{z}\left(1-k_{x} \frac{\bar{h}_{x}}{D a\left(1+\bar{h}_{x}^{2}\right)^{3 / 2}}\right) \Delta h=\left(1+\frac{k_{z}-k_{x} \bar{h}_{x}}{D a \sqrt{1+\bar{h}_{x}^{2}}}\right) \Delta C
$$

Combining together eqs. (44-47) and setting $\bar{h}_{x}=\cos \theta$ yields finally the following dispersion relation :

$$
\begin{aligned}
\omega_{h}= & -\frac{\left(-\bar{C}_{z}\right) k_{z}}{1+\left(\frac{k_{z}}{D a}\right)^{2}+2\left(\frac{k_{z}}{D a}\right) \cos \theta} \ldots \\
& \ldots\left\{\left[1+\left(\frac{k_{z}}{D a}\right) \cos \theta\left(\cos ^{2} \theta+\tan ^{2} \theta\right)\right] \ldots\right. \\
& \left.\ldots \pm j\left[\tan \theta+\left(\frac{k_{z}}{D a}\right) \cos \theta \sin \theta\left(\cos \theta+\left(\frac{k_{z}}{D a}\right)\right)\right]\right\}
\end{aligned}
$$

Remembering that $\cos \theta,-\bar{C}_{z}$, and $k_{z}$ are positive, one finds that $\Re e\left(\omega_{h}\right)$ is always negative, which means that any perturbation decreases, no matter what the local slope is. Both solutions, the flat and the curved one, are stable. 
Such an analysis would have to be modified in the presence of curvature effects, since they have been proved to destabilize CVD growth processes.

\subsection{Stability of singular points : Hamilton-Jacobi analysis}

\subsubsection{Displacement of individual points of the interface}

Consider the evolution of a surface in the case of oxidation $\left(\nu_{g}=\nu_{s}=-1\right)$, in vertical flux approximation.

To simplify notations, we will drop the primes corresponding to the moving coordinate frame. The local normal velocity $\mathbf{v}$ writes :

$$
\mathbf{v}=-D a C(h) \mathbf{n}=-D a \cdot \frac{1}{1+\frac{h D a}{\cos \theta}} \mathbf{n}
$$

The vector $\mathbf{v}$ is plotted at figure 7 for all possible values of $\theta$. Even though $\mathbf{v}$ is the local normal velocity of the surface, we will see that, for a point $\mathbf{M}$ of the surface, $\mathcal{W}=\frac{d \mathbf{M}}{d t} \neq \mathbf{v}$.

Since $\mathbf{v}$ and $\mathbf{n}$ are collinear, il will be practical to define the quantity :

$$
q=\mathbf{v} \cdot \mathbf{n}=-\|\mathbf{v}\|=-\frac{D a}{1+\frac{h D a}{\cos \theta}}
$$

and rewrite eq. (5) under a Hamilton-Jacobi form :

$$
\frac{\partial S}{\partial t}+q\|\nabla \mathbf{S}\|=\frac{\partial S}{\partial t}+\mathcal{H}=0
$$

If we suppose $\mathbf{v}$ to be differentiable, then the associated characteristic system is :

$$
\begin{aligned}
\frac{d x_{i}}{d t} & =\frac{\partial \mathcal{H}}{\partial\left(S_{x_{i}}\right)} \\
\frac{d\left(S_{x_{i}}\right)}{d t} & =\frac{-\partial \mathcal{H}}{\partial x_{i}}
\end{aligned}
$$


with $x_{i}=x, y, z$. After some algebra, eq. (53) may be rewritten in vector form :

$$
\begin{aligned}
\frac{d \mathbf{M}}{d t}=\mathcal{W} & =\nabla_{\mathbf{n}} q-\left(\nabla_{\mathbf{n}} q \cdot \mathbf{n}\right) \mathbf{n}+\mathbf{v} \\
& =\left(\mathbf{n} \wedge \nabla_{\mathbf{n}} q\right) \wedge \mathbf{n}+\mathbf{v}
\end{aligned}
$$

where $\nabla_{\mathbf{n}}=\frac{\partial}{\partial \mathbf{n}}$ is the (formal) derivative with respect to the local normal components.

To illustrate the calculus, we consider the $2 \mathrm{D}$ case. In our application, with $\mathbf{n}={ }^{t}(\sin \theta ; \cos \theta)$, one obtains :

$$
\left\{\begin{array}{l}
\mathcal{W}_{x}=q \sin \theta+\frac{\partial q}{\partial \theta} \cdot \cos \theta \\
\mathcal{W}_{z}=q \cos \theta-\frac{\partial q}{\partial \theta} \cdot \sin \theta
\end{array}\right.
$$

which gives, using eq. (51) :

$$
\left\{\begin{array}{l}
\mathcal{W}_{x}=-D a \frac{\cos ^{2} \theta \sin \theta}{(\cos \theta+h D a)^{2}} \\
\mathcal{W}_{z}=-D a \frac{\cos ^{3} \theta+h D a}{(\cos \theta+h D a)^{2}}
\end{array}\right.
$$

The graph of $\mathcal{W}$, noted $\Gamma_{\mathcal{W}}$, is also presented at figure 7 . This figure indicates that this graph may be constructed as the envelope of the set of lines defined by :

$$
\mathbf{M} \cdot \mathbf{n}=x \sin \theta+y \cos \theta=q(\theta)
$$

One notes that $\Gamma_{\mathcal{W}}$ shows three singular points. The first one lies at the bottom of the 
graph, for $\theta= \pm \frac{\pi}{2}$, and the other ones are for $\theta_{0}$ verifying :

$$
\cos ^{3} \theta_{0}-2 h D a+3 h D a \cos ^{2} \theta_{0}=0
$$

As shown at figure 8 , this angle belongs to the interval $\left.] \theta_{\lim } ; \pi / 2\right]$, where $\theta_{\lim }=\arccos (\sqrt{2 / 3})$ $\approx 35^{\circ} 44^{\prime}$, a value inferior to the angle domain corresponding to the vertical flux approximation.

\subsubsection{Application of Huygens' principle to the evolution of singular points}

The principle of the approach has been described in details in $[11,16]$. By analogy with the propagation of electromagnetic waves, the surface is assimilated to a wavefront, and it is stated that each point of the wavefront is a source of a secondary wave called Huygens wavelet. The evolving wavefront is defined as the common envelope of the wavelets. In the present case, the wavelets have no frequency (or an infinite one), so there is no phenomenon of interference, as in the electromagnetic application case.

Now, Huygens wavelets have to be defined. Continuing the analogy, one says that the wavelet is an elementary wavefront which is the locus of the points reachable by a source after a short time increment $\delta t$. This definition is equivalent to the approach described in section 4.2 .

So, the Huygens wavelet is the locus of the points defined by the vectorial equation (56). The evolved surface is then obtained as the common tangent envelope of the wavelets created by every point. An example of construction is given at fig. 9 .

For a local study, it will be considered that $h$ and $D a$ are fixed values, so that the Huyghens wavelet is of fixed size and shape in the vicinity of the studied point. Thus, applying the construction rules allows to discuss the stability of edges in the $\left(\theta_{-}, \theta_{+}\right)$ plane, as illustrated at fig. 10. It is possible to classify the singular points into four types, depending on the behavior : 
- Type I is a "sharp" reentrant edge, i.e. with two sides such as $\left|\theta_{i}\right|>\theta_{0}$. Its evolution leads to a smooth curve and two singular points noted $S^{\prime}$ on the scheme. Note that those evolved points are not of type I, so that type I edges are not stable.

- Type II is associated with reentrant edges with $\left|\theta_{i}\right|<\theta_{0}$ on one side only. A piece of smooth curve appears, and an evolved singular point $S^{\prime}$ is present. They may be considered as stable because the singular point is conserved.

- Type III edges are edges with small slopes : they evolve into smooth curves.

- Type IV edges are stable edges, marked with an $S$ on the figure : among them, one finds all salient edges.

The only edges which are present in a stationary curve are symmetrical edges, represented by the second diagonal of the $\left(\theta_{-}, \theta_{+}\right)$plane. By inspection of fig. 10 it is easy to see that

- Symmetrical type IV (salient) edges are stable ;

- Symmetrical type III edges are not stable and transform themselves into pieces of smooth curves ;

- Symmetrical type I edges are not stable : they split into two unsymmetrical type II edges which later on are prone to transform themselves into something else.

Accordingly, this confirms the fact that a stationary surface does not contain in practice any reentrant edge.

\section{$5 \quad$ Asymptotic limits}

It is easy to identify from the $D a \rightarrow 0$ and $D a \rightarrow \infty$ limits, the two asymptotic regimes that may be reached from the presented model. In the case of diffusion-limited ablation 
$(D a \rightarrow \infty)$, eq. (28) becomes :

$$
\frac{\partial h^{\prime}}{\partial t}=\frac{1}{h^{\prime}}-\frac{d \eta}{d t}
$$

from which the classical results on diffusion-limited etching or growth are recovered : the

interface is flat, and its velocity is not steady, unless $h^{\prime}$ becomes large. Considering $\frac{d \eta}{d t}=0$, there is a parabolic law $h^{\prime 2}=h^{\prime}(0)^{2}+t$.

The converse case makes the chosen reference time unusable, and one has to set $t_{0}^{*}=$ Da $t_{0}=\frac{h_{0}}{k\left(C_{0} v_{s}\right)}$. Note that $h_{0}$ is not any more a diffusion layer thickness. Indeed, the surface evolution does not depend any more on the distance to the chemical source.

Eq. (28) then becomes :

$$
\frac{\partial h^{\prime}}{\partial t^{*}}=\sqrt{1+\left(\frac{\partial h^{\prime}}{\partial x}\right)^{2}+\left(\frac{\partial h^{\prime}}{\partial y}\right)^{2}}-\frac{d \eta}{d t^{*}}
$$

Considering again $\frac{d \eta}{d t}=0$, one faces now a classical Hamilton-Jacobi equation, the solutions of which have been the object of a broad literature.

\section{Transient simulations}

The evolution equation for $S$ (equation 10-11) has been studied by a direct numerical integration. However, such an equation was obtained at first for a regular surface, and it has been seen that this was not necessarily the case. Moreover, in a discretized surface, every point can be singular, even if the continuous expression is differentiable. An explicit numerical scheme has been designed, using a discretized surface and applying eq. (56) to find the position of the evolved points and construct the new surface.

Once the surface is discretized, all points become singular. Their evolution, in the limit of locally constant $h$ and $D a$ parameters, is summarized at fig. 10; rules for the numerical 
scheme may be deduced from it.

The algorithm of surface construction is built as follows :

Step 1 : Discretization of the initial surface. Let $\delta x$ be a regular space increment and $(i, j)$ the discretized variables corresponding to $(x, t)$. The discretized surface is a set of points $\mathbf{M}_{i}^{j}={ }^{t}\left(x_{i}^{j}, z_{i}^{j}\right)$. To each segment $\left(\Sigma_{i}^{j}\right)=\left[\mathbf{M}_{i-1}^{j}, \mathbf{M}_{i}^{j}\right]$ is associated $\theta_{i}^{j}$, the angle between the normal and the vertical axis.

Step 2 : Evolution of adjacent segments At the time increment $j$, the point $\mathbf{M}_{i}^{j}$ is surrounded by two segments $\left(\Sigma_{i}^{j}\right)$ and $\left(\Sigma_{i+1}^{j}\right)$. As seen before, the evolved segments will obey to the following equations :

$$
\begin{array}{ll}
\left(\Sigma_{i}^{j+1}\right): & \sin \left(\theta_{i}^{j}\right)\left(x-x_{i}^{j}\right)+\cos \left(\theta_{i}^{j}\right)\left(z-z_{i}^{j}\right)=\mathcal{W}_{n}\left(\theta_{i}^{j}\right) \cdot \mathbf{n} \delta t \\
\left(\Sigma_{i+1}^{j+1}\right): & \sin \left(\theta_{i+1}^{j}\right) x+\cos \left(\theta_{i+1}^{j}\right) z-c_{2}=\mathcal{W}_{n}\left(\theta_{i+1}^{j}\right) \cdot \delta t
\end{array}
$$

where, $c_{1}=\sin \left(\theta_{i}^{j}\right) x_{i}^{j}+\cos \left(\theta_{i}^{j}\right) z_{i}^{j}$, and $c_{2}=\sin \left(\theta_{i+1}^{j}\right) x_{i}^{j}+\cos \left(\theta_{i+1}^{j}\right) z_{i}^{j}$.

The evolution of a type IV edge is given by the simple intersection of the evolved segments. For a type I,II or III edge, the evolution is given by the intersection of the evolved segments and the locus of points defined by $\mathcal{W}$ as seen in figures $10 \mathrm{~b}$ ).

Step 3 : Reconstruction Once the evolved surface has been built, there remains to choose new points $O(i, j+1)$ to represent it. In order to avoid point merging, we take $x_{i}^{j}=x_{i}$ constant $\forall j$.

With such a choice, we have :

- For a type II edge :

- if $\theta_{i}^{j}>\theta_{i+1}^{j}, z_{i}^{j+1}$ is given by the equation (64) with $x=x_{i}$; 
- if $\theta_{i}^{j}<\theta_{i+1}^{j}, z_{i}^{j+1}$ is given by the equation (63) with $x=x_{i}$;

- For a type I or III edge : $z_{i}^{j+1}=\mathcal{W}_{z}(\theta=0)$;

- For a type IV edge :

- if $\theta_{i}^{j}>\theta_{i+1}^{j}, z_{i}^{j+1}$ is given by the equation (63) with $x=x_{i}$;

- if $\theta_{i}^{j}<\theta_{i+1}^{j}, z_{i}^{j+1}$ is given by the equation (64) with $x=x_{i}$;

One of the most interesting points is to find out in what kind of situation a stationary solution made of circle arcs appears. An example is illustrated at fig.11 : an initial condition has been taken with an "inverse" profile, that is, with symmetrical singular points pointing towards the solid instead of the fluid, also featuring a curvature superior to the opposite of the stationary curvature. It is seen that relaxation towards the stationary "circle-arcs" solution occurs within a few dimensionless time units, with two phenomena $: i)$ the smooth arcs increase curvature, and a singular point appears, which then relaxes towards the stationary situation, and $i i$ ) the angle at the initial singular point (type I) diminishes rapidly and a smooth circle arc appears eventually.

\section{Application case : onset of cellular roughness from sublimation}

A numerical evaluation of the curvature radius $D a^{-1} h_{0}=D / k$ can be made, taking typical values of plasma jet conditions, such that the surface temperature is about $4300 \mathrm{~K}$ and the pressure approximately $5 \mathrm{MPa}$. In such conditions, the major carbon-containing species is $C_{3}$ [1]. The value of the sticking coefficient is known experimentally $[17,18]: \alpha \simeq 0.028$. It is considered that, even if the flow is turbulent, there exists a thin layer close to the wall where laminar flow occurs ; this layer has to be traversed by the gaseous species, so the diffusion coefficient in laminar flow is the critical transport property. Its value can be estimated by the classical theory of Chapman-Enskog [19], provided the parameters of interaction. Here the data of [20] has been chosen : a Lennard-Jones potential $(\sigma=$ 
$0.445 \mathrm{~nm}$ and $\left.\frac{\epsilon}{k_{B}}=128 \mathrm{~K}\right)$. These values lead to a diffusion coefficient $D=0.026 \mathrm{~cm}^{2}$. $s^{-1}$. Then the resulting value for the curvature radius is about $3 \mu \mathrm{m}$. This value scales reasonably with the features of fig. 3, provided the large uncertainty on the sticking coefficient. In fact, measuring this scale can be a way to have an indirect assessment of $\alpha$.

A remark has to be done about such a scale. If the heat flux is strongly varied, the fact that sublimation is the main ablation cause renders it "robust", because flux variations will primarily lead to changes in sublimation flux, but not in surface temperature ; since $D / k$ is mainly a function of $T$, it will not vary strongly when the heat flux varies.

It is also straightforward to evaluate the possible importance of curvature effects, as depicted in $[8,9]$. The correction that has to be added to the source term is :

$$
R^{\prime}=R(1+\Gamma \kappa)
$$

where $\kappa$ is the curvature and $\Gamma=\frac{\gamma_{f s} v_{s}}{\mathcal{R} T}$ is the length scale for the correction. Such a $\Gamma$ factor ranges between $10^{-10}$ and $10^{-8} \mathrm{~m}$ depending on the chosen value for the surface tension of carbon. It is thus clear that the curvature correction is negligible in the presented case.

Now a question remains : if the identified scale is micrometric, how to explain the millimeter-scale features of fig. 1b) ? An answer, at least partial, may arise from the consideration of heat transfer by diffusion.

\section{Thermal conduction scalloping}

Let us now consider that the transported quantity that brings a rate limitation is temperature instead of gas species mass. We are working now at a length scale that is superior to 
the mass-transfer boundary layer, and may consider that there is an effective rate constant

$$
k^{\star}=R / C_{0}=\left(k^{-1}+h_{0} D^{-1}\right)^{-1}
$$

that incorporates the effect of gas diffusion through this layer. On the other hand, it will be assumed that $k^{\star}$ depends on $T$ through, for example, an Arrhenius law. Summarizing, the reaction rate is written under the form :

$$
R=k^{\star} C_{0}=A \exp \left(-\frac{E_{a}}{\mathcal{R} T}\right) C_{0}
$$

By analogy with the mass transfer case, it will be considered that the temperature is held constant at a certain height :

$$
T(z=\eta)=T_{0}
$$

Applying again the hypothesis of vertical flux approximation, the heat flux is given by :

$$
q_{z}=-\frac{\lambda}{h^{\prime}}\left(T(z=h)-T_{0}\right)
$$

In addition to this, let us assume that there is a constant, known, vertical heat flux at the top of the thermal boundary layer $q_{z}(z=\eta)=q_{0}$, and that the thermal conductivity is not a function of temperature (indeed, it depends on temperature much less than the reaction rate). Then, the temperature will be a simple function of $h^{\prime}$ :

$$
T\left(h^{\prime}\right)=T_{0}-\frac{q_{0}}{\lambda} h^{\prime}
$$

On the other hand, recalling eqs. $(1-3)$ and (17) as well as the definition of $\theta$, one has :

$$
\mathcal{V}=\frac{\partial h}{\partial t}=R(T(h(x))) \cos \theta
$$

The stationarity of the profile implies, as before, that the velocity is independent of the 
$x$ coordinate. This yields the following result :

$$
\kappa=\frac{1}{\sqrt{1+\left(\frac{\partial h}{\partial x}\right)^{2}}}\left(\frac{\partial h}{\partial x}\right)^{-1} \frac{\partial \ln R}{\partial x}
$$

Making use of eqs. (68) and (66) finally gives :

$$
\kappa=-\frac{E_{a}}{\mathcal{R} T(h(x))} \cdot \frac{q_{0}}{\lambda T(h(x))} \cos \theta
$$

Choosing a reference length $h_{0}=\frac{\lambda T_{0}}{q_{0}}$ gives the following relation :

$$
\tilde{\kappa}=-\gamma_{a} h_{0}^{-1}\left(1-\tilde{h}^{\prime}\right)^{-2} \cos \theta
$$

where $\gamma_{a}$ is the scaled activation energy $E_{a} / \mathcal{R} T_{0}$. In this solution, it is found that the curvature radius is not any more a constant, but a function of the depth $h^{\prime}$. Fig. 12 is a plot of a numerical evaluation of $h^{\prime}(x)$, where it is seen that the shape differs neatly from the semi-circle in the region of high slopes. In practice, the dimensionless depth is no more than 0.1 , so that the surface temperature is $90 \%$ of the top fluid temperature.

This suggests that the maximal order of magnitude for the scallop curvature radius is $\frac{h_{0}}{0.2 \gamma_{a}}$. Numerical application in the case of fig. 1b) is the following : $q_{0} \approx 10 \mathrm{MW} \cdot \mathrm{m}^{-2}$, $T_{0} \approx 4800 K, \lambda_{0} \approx 10 \mathrm{~W} \cdot \mathrm{m}^{-1} \cdot K^{-1}, \gamma_{a} \approx 12$. Then $r \approx h_{0} / 2.4 \approx 16 \mathrm{~mm}$. This matches approximately the observed scale.

Fortunately, the fact that this length scale is much higher than the preceding one helps to validate the isothermal hypothesis that has been given in the case of mass diffusional limitations. 


\section{Conclusion}

The question of roughness set-up due to ablation in atmospheric reentry has been addressed. Apart from the trivial cause due to the material inhomogeneities, another cause seems to act even in the case of homogeneous materials. It is basically a dynamical effect based on the concurrence between transfer and reaction : when the surface is higher, the consumption rate is higher, but when it is more inclined with respect to the principal transfer direction, the rate is lowered. Thus, points with different altitudes may well have the same recession velocity, leading to a rough surface profile.

A simplified, isothermal model featuring the interaction of 1D (normal) diffusion with heterogeneous reaction or sublimation has been built, and it shows that in the intermediate regime (that is, neither diffusion-limited nor reaction-limited), cellular roughness may appear, under the form of a stable surface made of circle arcs in 2D (and by extension of sphere caps in 3D). Such a morphology has been indeed found experimentally in various cases. One of the successes of the presented model is that it helps understand why a characteristic length scale, different from the turbulence-related scales, appears in ablation, even without any heterogeneity of the substrate. Also, since this roughness is capable of being formed in laminar flow conditions, it is a new candidate to the explanation of laminar-to-turbulent transition in the flow regime around the reentry body.

Two length scales have been found for the curvature radii of the roughness features : the first one, arising from mass transfer, is much smaller than the second one, arising from heat transfer. Indeed, both of them are known experimentally.

Consequences of such a behavior have to be carefully considered with respect to other experimental results, and the solidity of the model with respect to addition of new physical features, such as the curvature-enhanced chemical reactivity or an explicit coupling 
between mass, heat and momentum transfers, has to be tested in future work. Also, extensions of the simple model to anisotropic and composite surfaces will be treated in other papers.

\section{Acknowledgements}

The authors wish to thank D. Rousselle (CEA) for transmitting profilometry data, which has been obtained at Laboratoire de Microanalyse des Surfaces (ENSMM Besançon), as well as J. Lachaud (LCTS) for providing micrograph at figs. 2 and 3. Snecma Propulsion Solide is acknowledged for Masters and $\mathrm{PhD}$ grants to Y. A. 


\section{References}

[1] G. Duffa, Ablation, Monograph ISBN 2-7272-0207-5, CEA - CESTA, Le Barp, France (November 1996).

[2] M. D. Jackson, Roughness induced transition on blunt axisymmetric bodies, Interim Report SAMSO-TR-74-86, Vol. 15, Passive Nosetip Technology (PANT) Program (April 1974).

[3] D. C. Reda, Correlation of nosetip boundary-layer transition data measured in ballisticrange experiments, Report SAND 79-0649, Vol. 10, Sandia (November 1979).

[4] M. R. Wool, Summary of experimental and analytical results, Interim Report SAMSO-TR74-86, Vol. 10, Passive Nosetip Technology (PANT) Program (January 1975).

[5] C.-C. Hwang, H.-Y. Yang, J.-Y. Hsieh, Y.-M. Dai, Three-dimensional morphological instabilities in chemical vapor deposition films, Thin Solid Films 304 (1997) 371-380.

[6] B. J. Palmer, R. G. Gordon, Local equilibrium model of morphological instabilities in chemical vapor deposition, Thin Solid Films 158 (1988) 313-341.

[7] B. J. Palmer, R. G. Gordon, Kinetic model of morphological instabilities in chemical vapor deposition, Thin Solid Films 177 (1989) 141-159.

[8] D. J. Srolovitz, A. Mazor, B. G. Bukiet, Analytical and numerical modeling of columnar evolution in thin films, J. Vac. Sci. Technol. A 6 (4) (1988) 2371-2380.

[9] J. J. Thiart, V. Hlavacek, H. J. Viljoen, Chemical vapor deposition and morphology problems, Thin Solid Films 365 (2) (2000) 275-293.

[10] E. Dourthe, A. Cosculluela, D. Rousselle, Etude des propriétés d'ablation des graphites à l'aide d'une torche à plasma, Report DETN/MA576/95-DR, CEA (1995).

[11] I. V. Katardjiev, A kinematic model of surface evolution during growth and erosion : Numerical analysis, J. Vac. Sci. Technol. A 7 (6) (1989) 3222-3232. 
[12] C. W. Hirt, B. D. Nichols, Volume of fluid (VOF) method for the dynamics of free boundaries, J. Comput. Phys. 39 (1981) 201-225.

[13] I. C. Lin, L. K. Sproul, M. Olmos, An aerothermal model for ablation heat shields, AIAA paper (94-0247).

[14] J. Nagle, R. F. Strickland-Constable, Oxidation of carbon between 1000-2000 ${ }^{\circ} \mathrm{C}$, in: Proceedings of $5^{\text {th }}$ Conference on Carbon, Pergamon Press, 1962, pp. 154-164.

[15] R. W. Schrage, A Theoretical Study of Interphase Mass Transfer, Columbia University Press, New York, 1953.

[16] I. V. Katardjiev, The application of the Huyghens principle to surface evolution in inhomogeneous, anisotropic and time-dependent systems, J. Phys. D: Appl. Phys. 22 (1989) $1813-1824$.

[17] H. B. Palmer, M. Shelef, Vaporization of carbon, in: P. L. W. Jr. (Ed.), Chemistry and Physics of Carbon, Vol. 4 of Chemistry and Physics of Carbon, Marcel Dekker, New York, 1968, pp. 85-135.

[18] R. L. Baker, M. A. Covington, The high temperature thermochemical properties of carbon, Interim Report SD-TR-82-19, Office of Naval Research (March 1982).

[19] J. O. Hirschfelder, C. F. Curtiss, R. B. Bird, Molecular theory of gases and liquids, 2nd Edition, John Wiley \& sons, 1963.

[20] L. Biolsi, J. Fenton, B. Owenson, Transport properties for a mixture of the ablation products $C, C_{2}$ and $C_{3}$, in: T. E. Horton (Ed.), Thermophysics of Atmospheric Entry, Vol. 82 of Progress in Astronautics and Aeronautics, AIAA, New York, 1982. 


\section{Table Captions}

- Table 1. Balance equations, stoichiometric coefficients, and boundary conditions for two causes of ablation. 


\section{Tables}

\begin{tabular}{|c|c|c|c|c|c|c|}
\hline & Type & Balance & $\nu_{s}$ & $\nu_{g}$ & $\mathrm{BC}$ at top & $\mathrm{BC}$ at interface \\
\hline 1 & Oxidation & $C(s)+O_{2}(g) \rightarrow 2 C O(g)$ & -1 & -1 & $C(z=\eta)=1$ & $\mathbf{J} \cdot \mathbf{n}=\nu_{g} k C$ \\
\hline 2 & Sublimation & $n C(s) \rightleftharpoons C_{n}(g)$ & $-n$ & +1 & $C(z=\eta)=0$ & $\mathbf{J} \cdot \mathbf{n}=\nu_{g}(\alpha\langle c\rangle / 4)(\bar{C}-C)$ \\
\hline 3 & Deposition & $C_{n} H_{m}(g) \rightarrow n C(s)+\frac{m}{2} H_{2}(g)$ & $+n$ & -1 & $C(z=\eta)=1$ & $\mathbf{J} \cdot \mathbf{n}=\nu_{g} k C$ \\
\hline
\end{tabular}

Table 1

Balance equations, stoichiometric coefficients, and boundary conditions for two causes of ablation. Comparison with CVD 


\section{Figure Captions}

- Figure 1. Profilometry maps of samples of a polycristalline graphite, ablated in laminar (top) and turbulent (bottom) regime.

- Figure 2. SEM micrograph of an ablated $C / C$ sample (ex-phenolic resin/carbon composite)

- Figure 3. SEM micrograph of ablation scallops on a $C / C$ composite fiber.

- Figure 4. Domain and notations for the model.

- Figure 5.Representation of a possible steady, nontrivial solution of the ablation profile in isothermal vertical flux approximation.

- Figure 6. Representation of the concentration field for the nontrivial solution of the ablation profile (fig. 5).

- Figure 7 Polar plot of normal velocity $\mathbf{v}$ and velocity of an interface point $\mathcal{W}$. For this plot, $h=1$ and $D a=1$.

- Figure 8. Critical angle $\theta_{0}$ vs. h.Da.

- Figure 9. Example of construction for a surface.

- Figure 10. Edge evolution diagram. The initial edge is defined by the angle $\theta_{+}$and $\theta_{-}$ as indicated in the sketch at the top of the figure ; the edge evolution after a time $\delta t$ is drawn in the $\left(\theta_{+}, \theta_{-}\right)$plane. The solutions are symmetrical with respect to the second principal diagonal of the plane.

- Figure 11. An example of transient behavior, starting from an "inverted curvature" initial solution.

- Figure 12. Dimensionless plot of an ablation scallop from the heat-transfer model. 


\section{Figures}

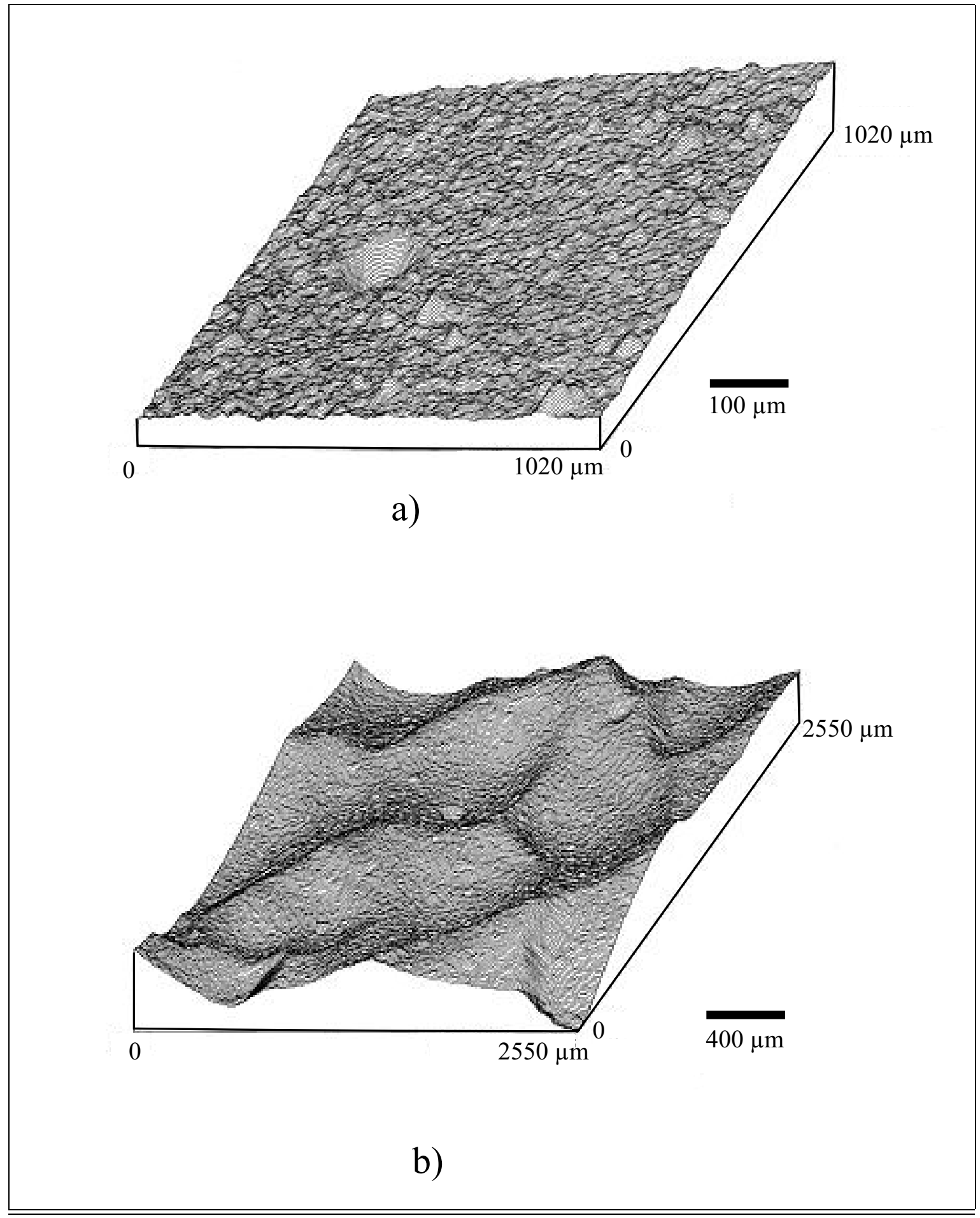

Fig. 1. Profilometry maps of samples of a polycristalline graphite, ablated in laminar (top) and turbulent (bottom) regime. 


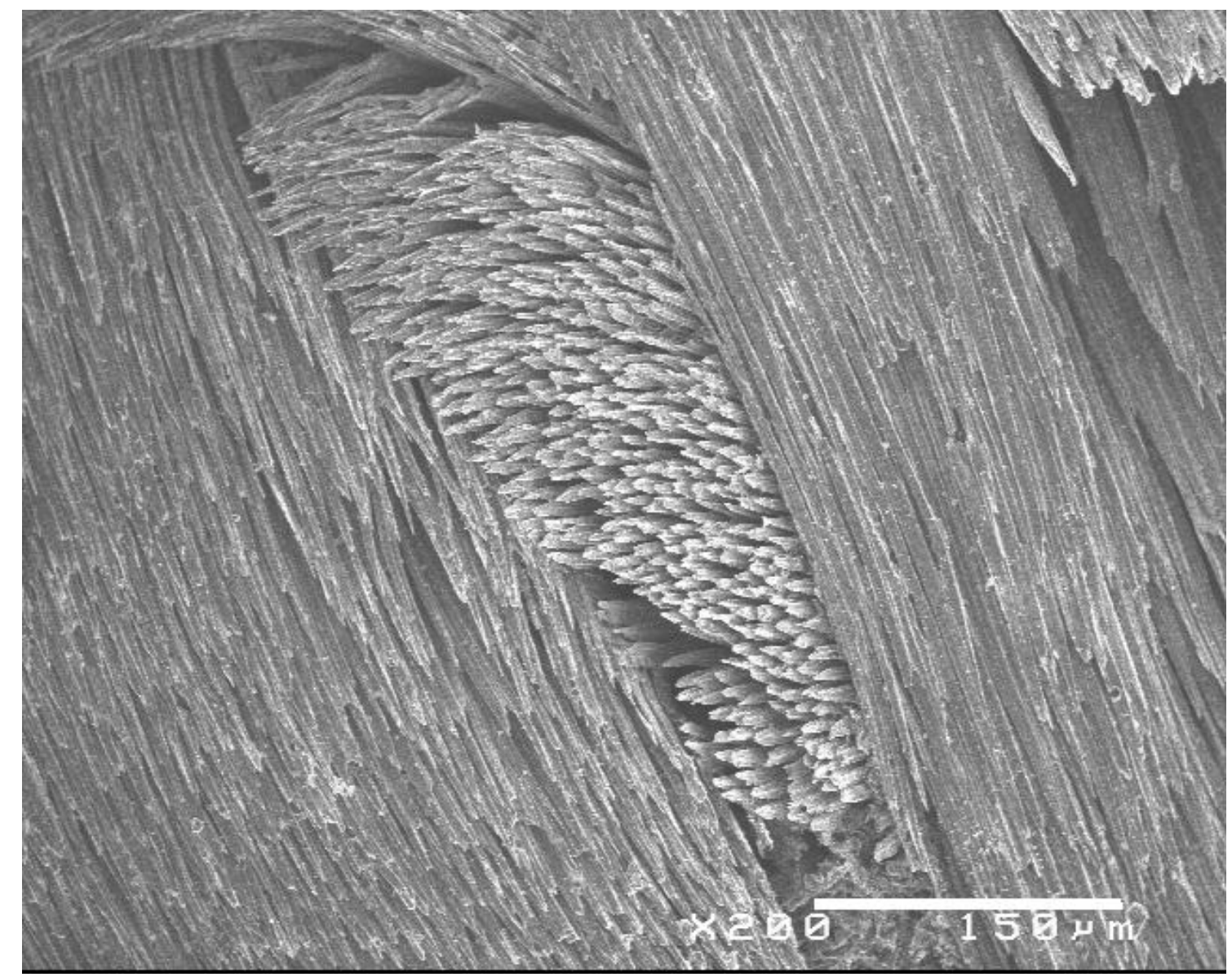

Fig. 2. SEM micrograph of an ablated $C / C$ sample (ex-phenolic resin/carbon composite) 


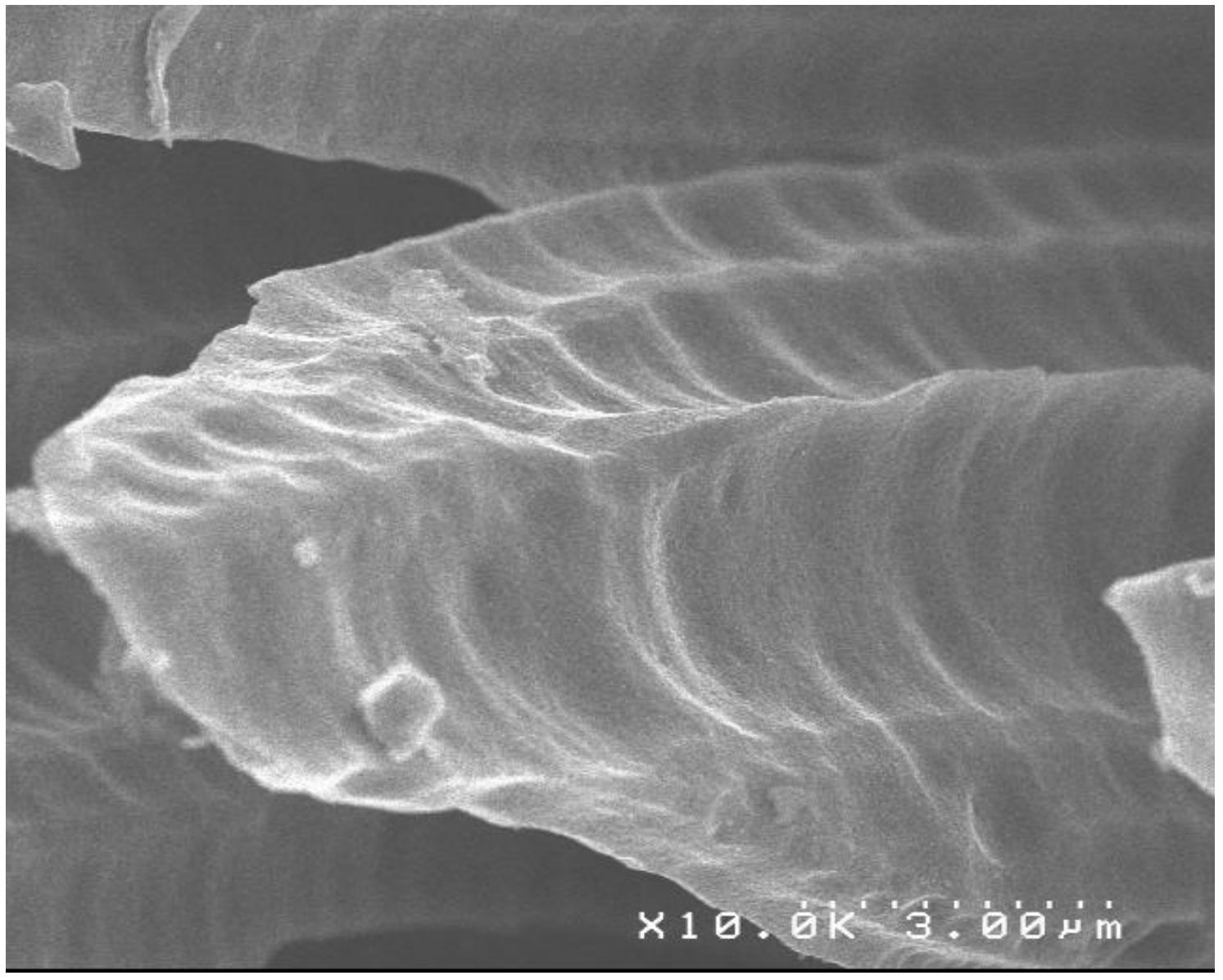

Fig. 3. Ablation scallops on a $C / C$ composite fiber. 


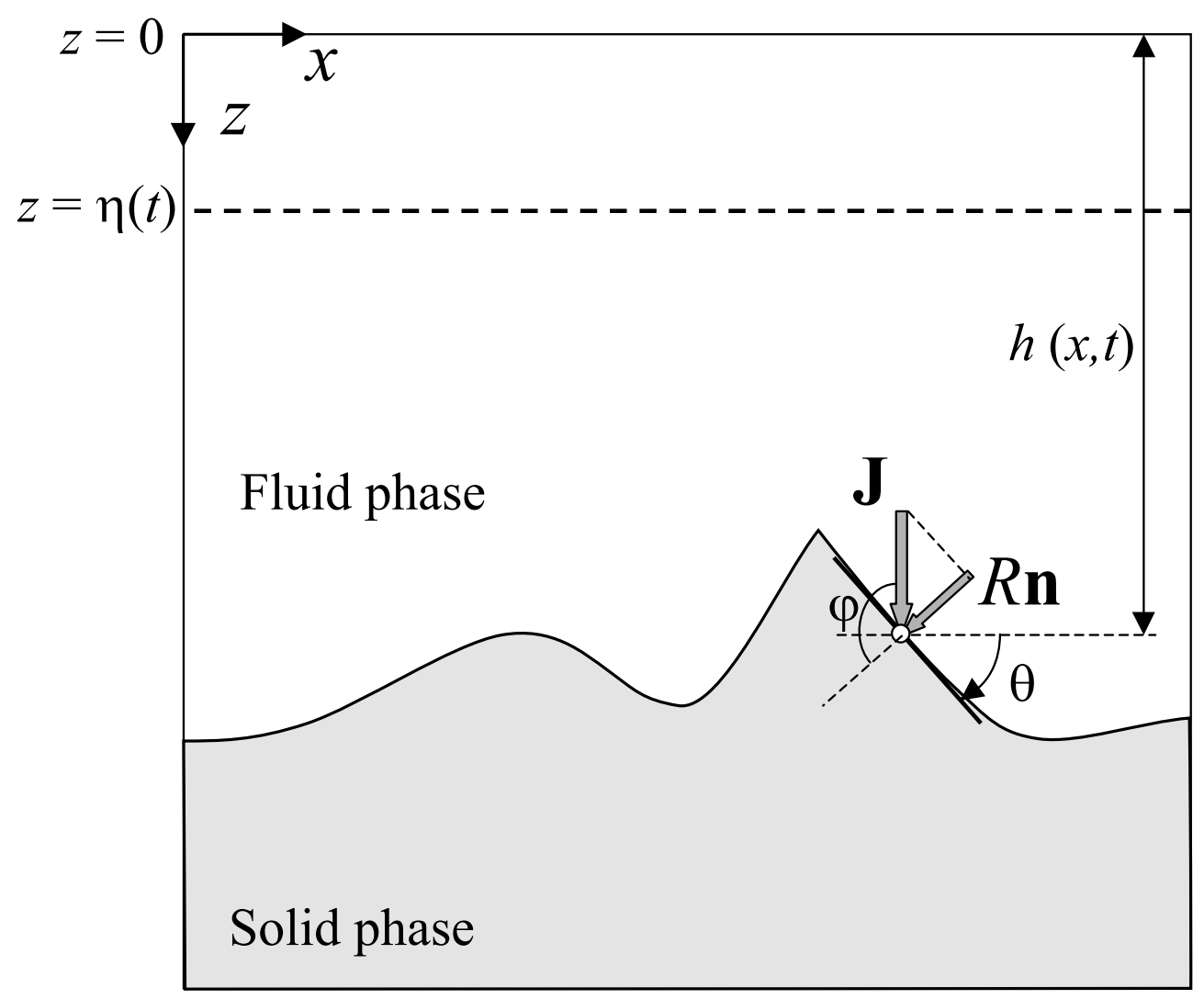

Fig. 4. Domain and notations for the model 


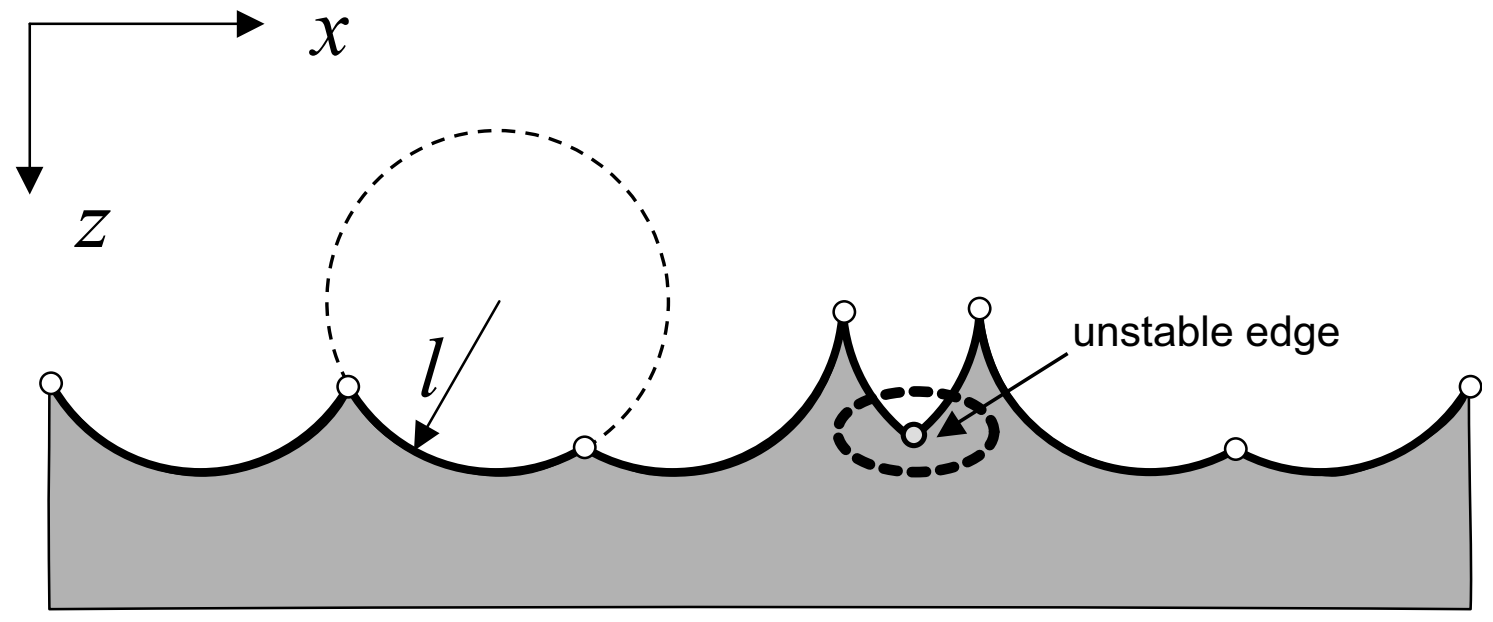

Fig. 5. Representation of a possible steady, quasi-1D, nontrivial solution of the ablation profile 


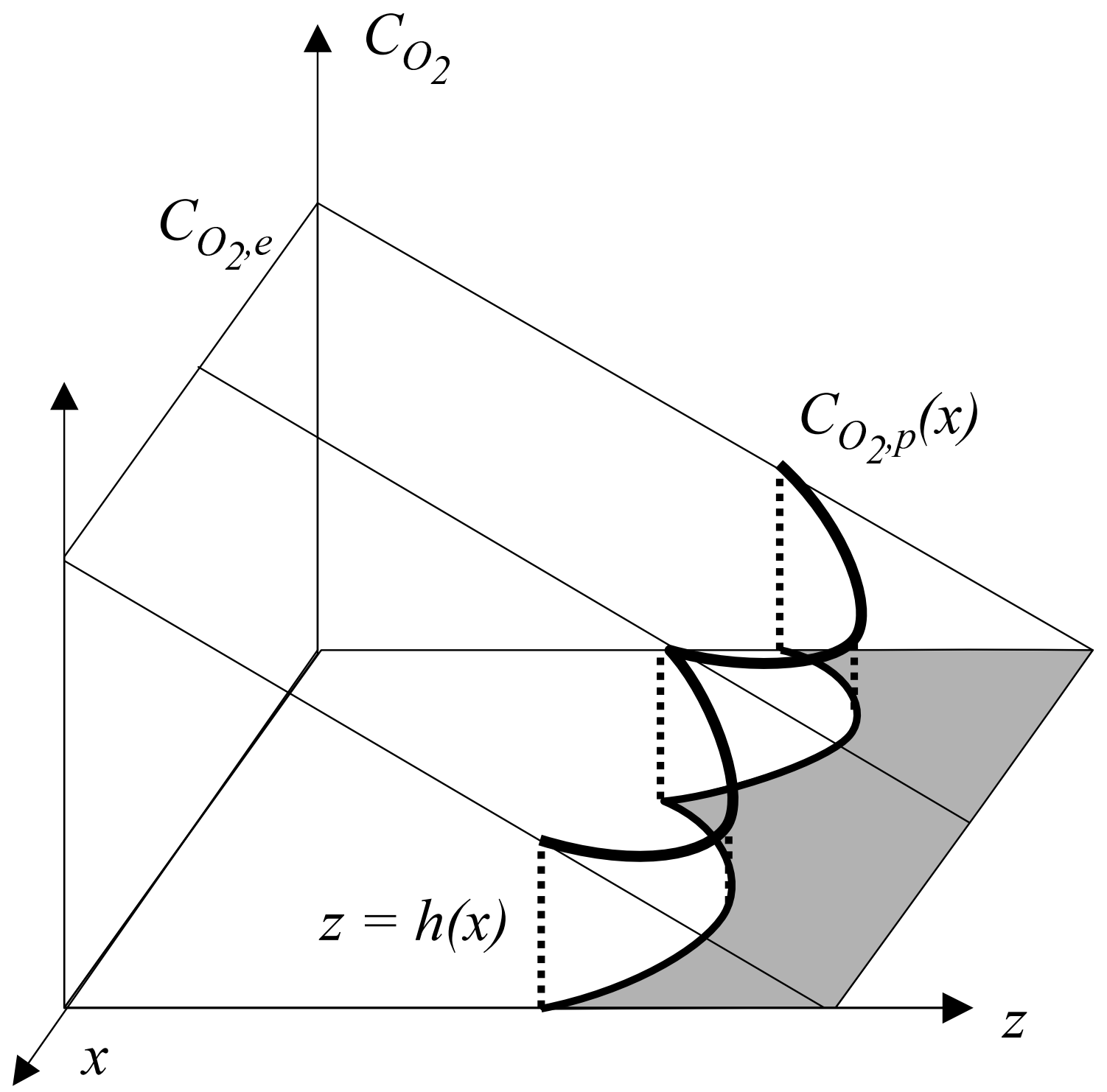

Fig. 6. Representation of the concentration field for a steady, quasi-1D, nontrivial solution of the ablation profile 


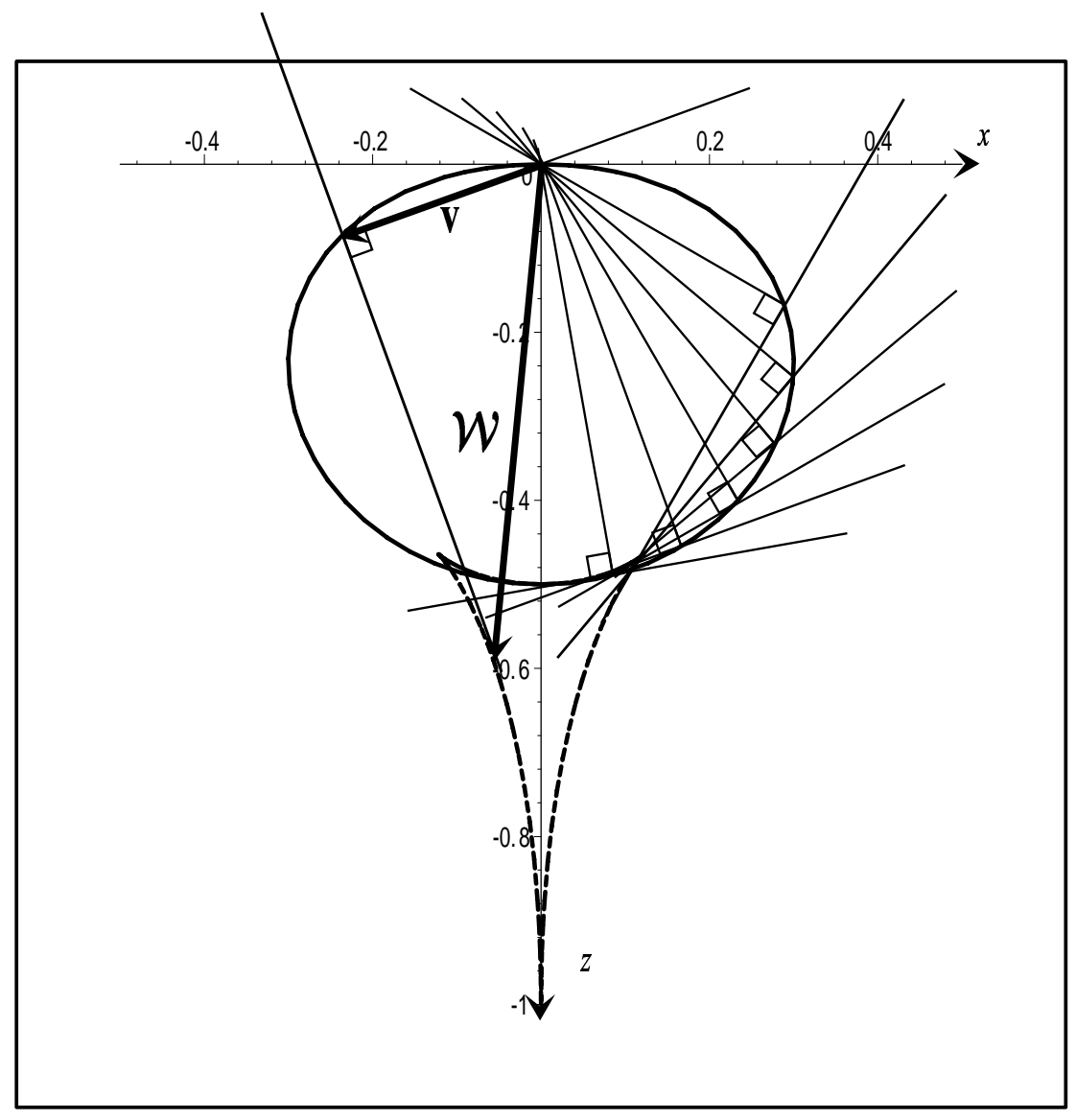

Fig. 7. Parametric plot of $\mathbf{v}$ and $\mathcal{W}$. The parameters $D a$ and $h$ have been set to unity. 


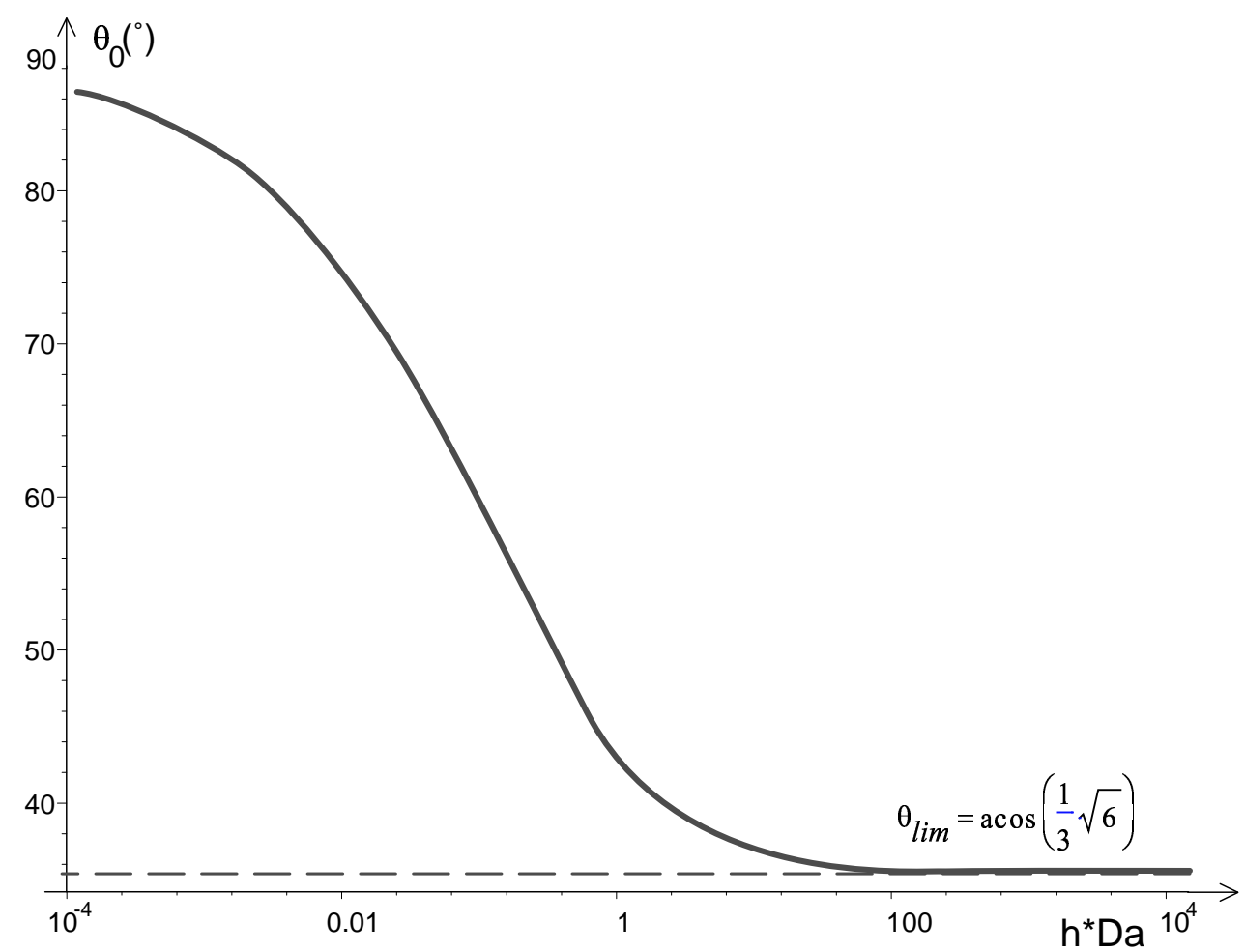

Fig. 8. Critical angle $\theta_{0}$ vs. $h * D a$. 


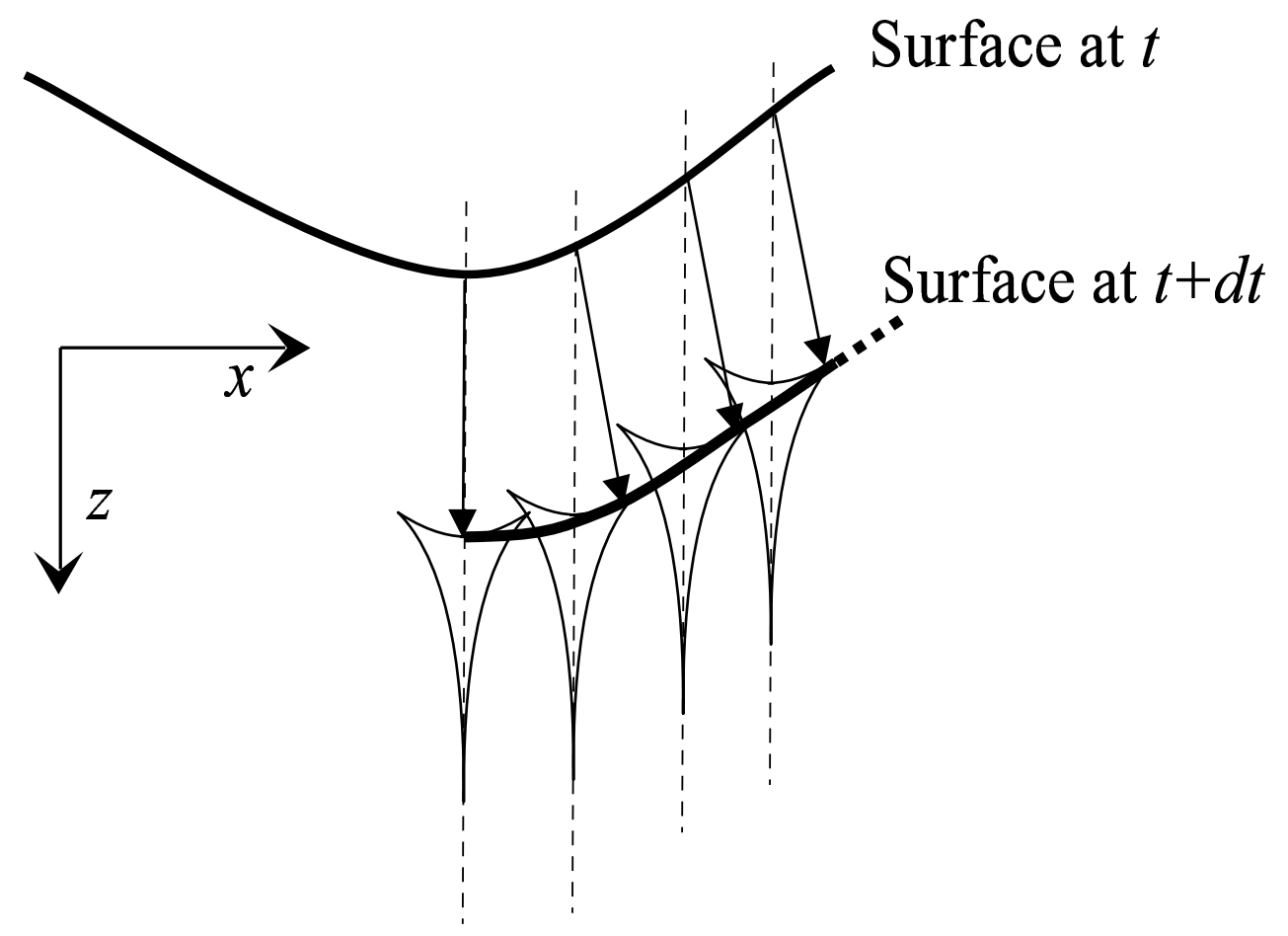

Fig. 9. Example of construction of a surface. 


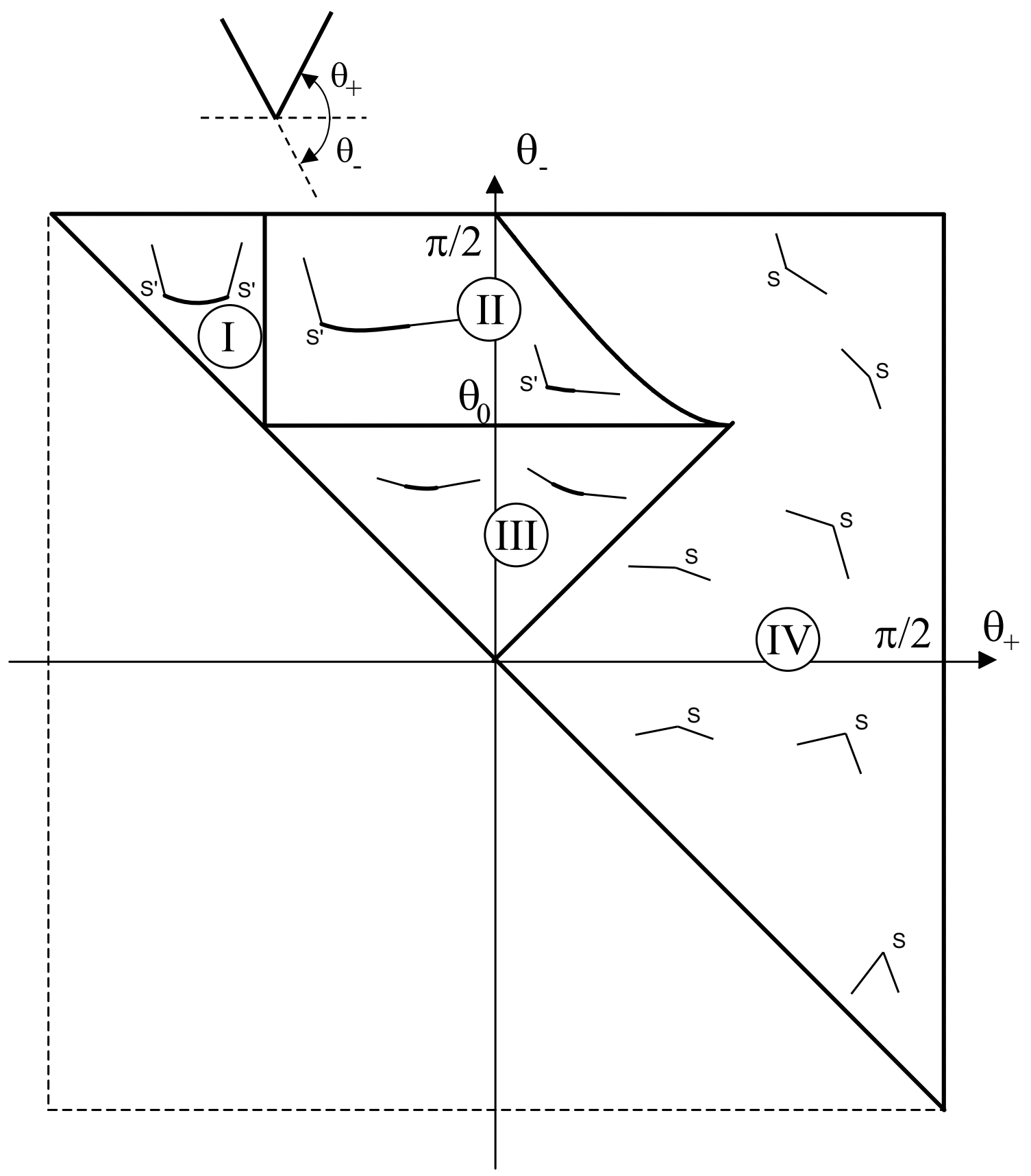

Fig. 10. Evolution of edges. 


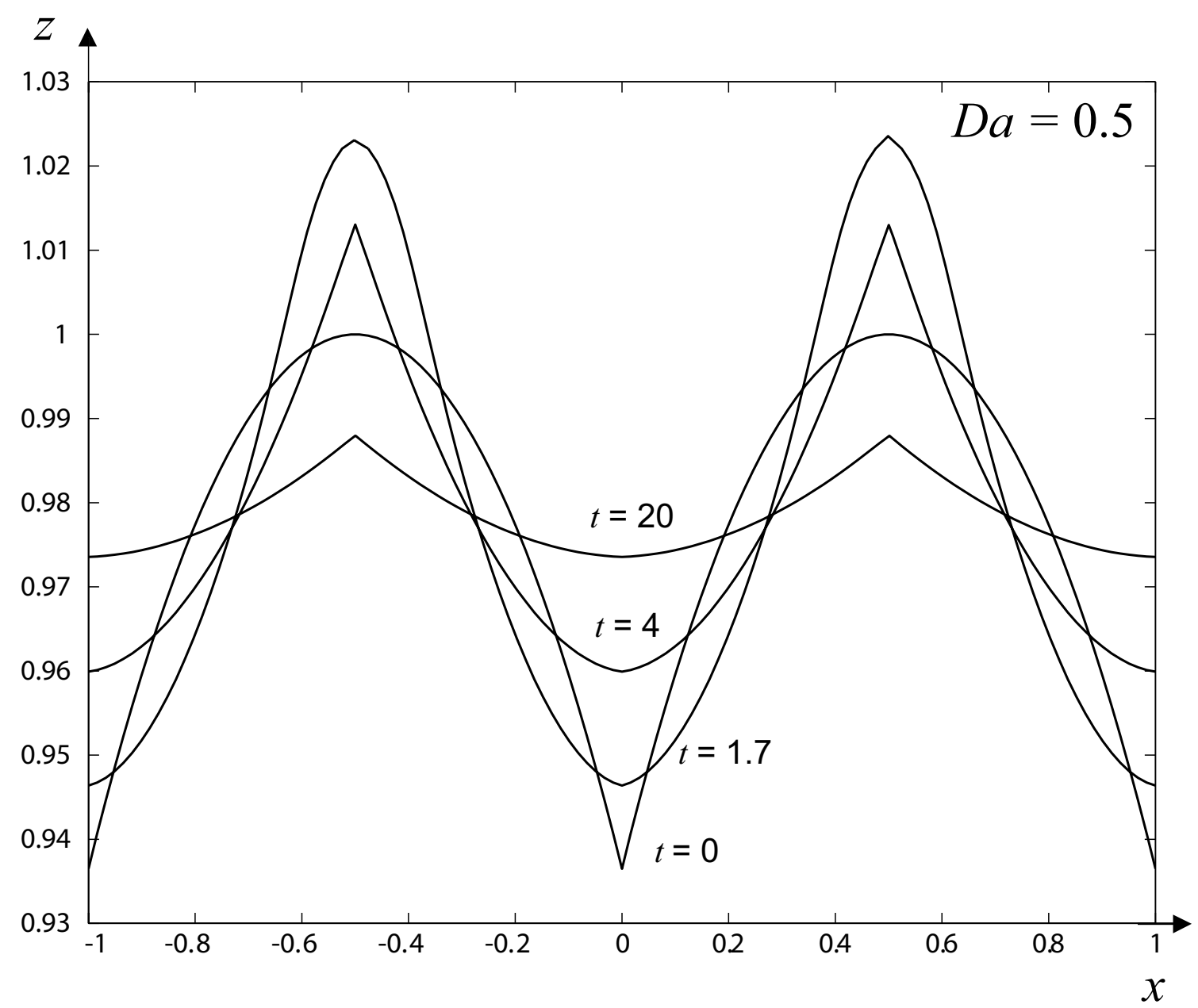

Fig. 11. An example of transient simulation. 


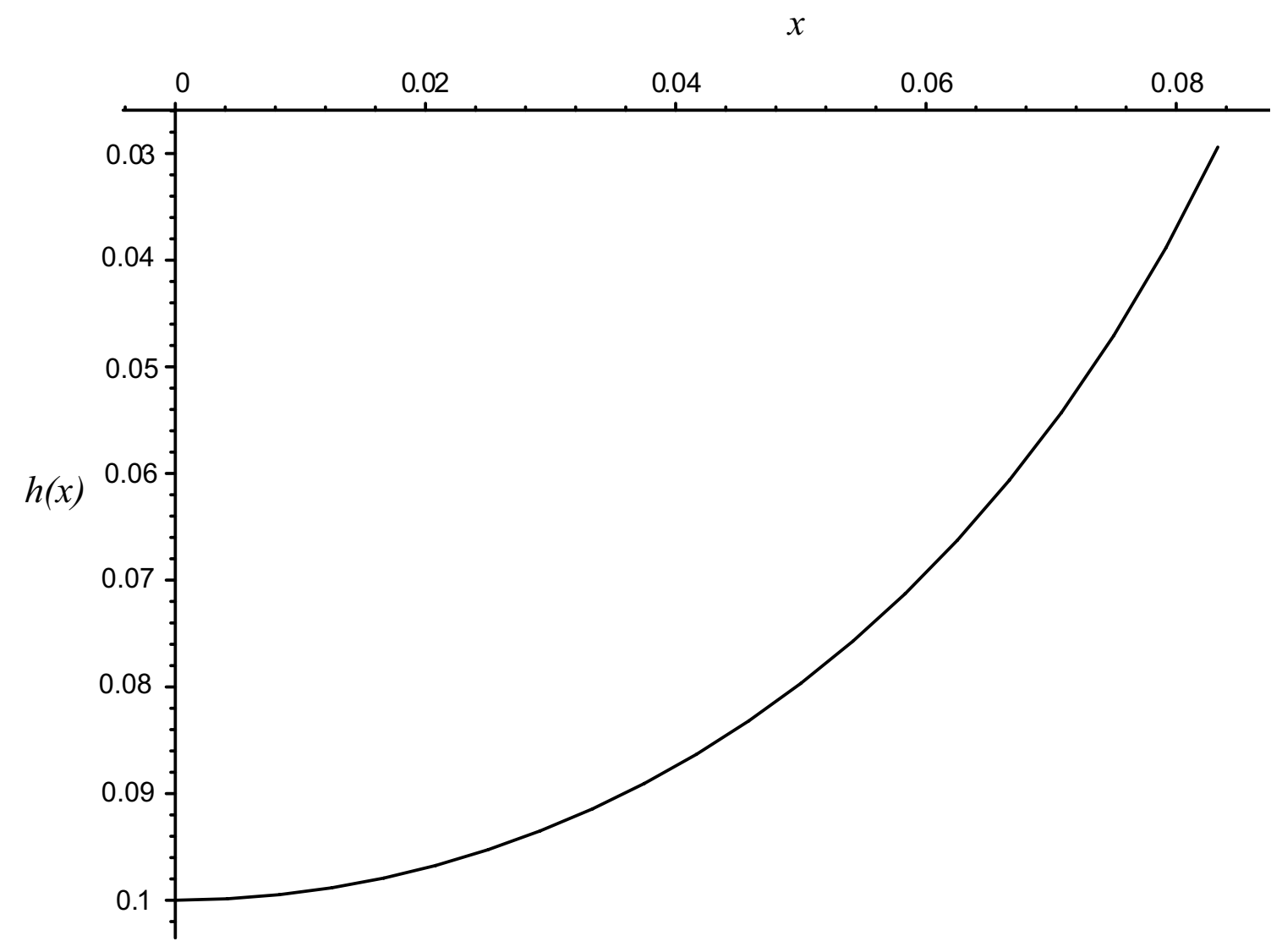

Fig. 12. Dimensionless plot of a thermal scallop evaluated numerically. Here $\gamma=12$, and the reference length is given by heat conduction $h_{0}=\lambda_{0} T_{0} q_{0}^{-1}$. 